Article

\title{
Appraisal of Genetics and Heterosis of Important Traits in Chilli Pepper Cultivated under the Influence of Chilli Leaf Curl Virus Disease
}

\author{
Vijeth S1, Sreelathakumary I', Rafeekher $\mathbf{M}^{2}$ and Prashant Kaushik 3,4,* \\ ${ }^{1}$ Department of Vegetable Science, College of Agriculture, Kerala Agricultural University, Thrissur, Kerala 680656, \\ India \\ 2 Department of Pomology and Floriculture, College of Agriculture, Kerala Agricultural University \\ 3 Instituto de Conservación y Mejora de la Agrodiversidad Valenciana, Universitat Politècnica de València, 46022 \\ Valencia, Spain \\ ${ }^{4}$ Nagano University, 1088 Komaki, Ueda, Nagano 386-0031, Japan \\ * Correspondence: prakau@doctor.upv.es; Tel.: +34-963-877000
}

\begin{abstract}
Chilli leaf curl virus (ChiLCV) is a severe threat to the successful cultivation of the chilli peppers. Besides, the genetics and heterosis for important traits under the influence of ChiLCV infestation is not well studied. Therefore here, were crossed seven genotypes (lines) with high yield and quality attributes with four highly resistant genotypes (testers) in a line $(\mathrm{L}) \times$ tester $(\mathrm{T})$ mating design to produce $28 \mathrm{~F}_{1}$ hybrids. Parents and their hybrids were evaluated for yield attributes and ChiLCV resistance. All the four testers were symptomless and among seven lines, two were moderately resistant and five were moderately susceptible to the ChiLCV. Among 28 F1 hybrids, 12 showed a moderate resistant reaction, 11 were moderately susceptible and five susceptible to the ChiLCV disease. Based on per se performance, most promising hybrid combinations were $\mathrm{L} 3 \times \mathrm{T} 2$, $\mathrm{L} 6 \times \mathrm{T} 1$, and $\mathrm{L} 3 \times \mathrm{T} 1$ for yield attributes. Whereas the superior crosses for ChiLCV resistance based on heterobeltosis, standard heterosis and specific combining ability test (SCA) effects were L6 $\times$ T1 and L7 $\times$ T3. Lines and testers showed significant differences for all the characters except for the plant height. The general combining ability (GCA) effects for testers were significant for all the traits except for days to first harvest. The ratio of $\sigma^{2} \mathrm{GCA} / \sigma^{2} \mathrm{SCA}$ indicated the predominance of non-additive gene effects in the inheritance of traits studied in this experiment. The contribution of lines was more compared to testers for all the characters except for primary branches plant 1. Overall, this study provides valuable information regarding the genetics and heterosis in chilli with respect to ChiLCV infestation.
\end{abstract}

Keywords: chilli pepper; ChiLCV; GCA; SCA; Line × Tester

\section{Introduction}

Chilli (Capsicum spp.) is a major vegetable and spice crop of family Solanaceae. The genus Capsicum comprises of at least 34 wild species, and five species viz., Capsicum annuum, C. frutescens , C. chinense, C. pubescens and C. baccatum (Linnaeus) have been domesticated and cultivated [1]. Most of the cultivated and wild species of Capsicum have chromosome number $2 \mathrm{n}=2 \mathrm{x}=24$. C. annuum $\mathrm{L}$. is the most cultivated species throughout the world, and they are categorised as sweet pepper /or bell pepper (non-pungent fruits) and hot pepper /or chilli pepper (pungent fruits [2,3]. Chilli pepper is widely grown as a spice crop 
in the tropical and temperate region, whereas sweet pepper is cultivated as high-value greenhouse crop [4]. Chilli is native to Central and South America, and India is considered as the secondary centre of diversity for $C$. annuum [5]. The outcrossing in chilli ranges from $7-65 \%$ under field conditions, therefore considered as facultative cross-pollinating species [6,7].

Moreover, chilli pepper is the second largest commodity after black pepper in international spice trade on economic terms. Chilli pepper is used in lots of forms, which include fresh or cooked vegetables, spices or herbs and as numerous processed products [8]. Chilli pepper is a vital source of vitamins (A, C and E) and minerals (potassium, magnesium and iron) [9]. During recent years there is an increase in productivity of chilli pepper due to the cultivation of high yielding and disease resistant $\mathrm{F}_{1}$ hybrids rather than the open-pollinated cultivars. Moreover, fruit yield in chilli has been increased by $35-50 \%$ due to heterosis breeding [10]. In the latest years, chilli hybrids have become very popular with the farmers due to their superior per se performance. Chillies grown from hybrid seeds are uniform in maturity as well as high in yield [11]. The superior performance of hybrids is manifested because of better plant vigour, high growth and development, earliness, increased productivity and higher degrees of resistance to biotic and abiotic stresses [12].

Globally, more than 35 viruses have been reported under natural conditions. Among them, the Chilli leaf curl disease (ChiLCD) caused by geminivirus, namely, Chilli leaf curl virus (ChiLCV) and transmitted by the whitefly (Bemisia tabaci G.) is one of the severe production constraints of chillies globally [13]. The disease inflicts both the quantitative and the qualitative yield losses which often reach 100 percent. The disease appears in epidemic form in autumn season in North Indian plains and during the summer season in South India. The characteristic symptoms of ChiLCD include upward curling, reduced size of leaves, puckering, stunted growth with no flowers and fruits in severely affected plants [14]. A recent survey and molecular characterization of chilli infecting virus revealed that a new begomovirus species, namely, Chilli leaf curl Vellanad virus (ChiLCVV) is responsible for ChiLCD in Kerala, India [15].

Diverse cultural and chemical tactics were attempted to manage the disease without plenty of achievements. Managing the disease with pesticides has been a hard challenge because of recurrent development of resistance against pesticides by whitefly. The exploitation of host-plant resistance is a safe, durable and economical approach to manage the disease [16]. Availability of resistant donor(s) is a prerequisite for any resistance breeding programme. In general, wild relatives or accessions of the cultivated species are renowned for their wealth of useful genes including those for disease-resistant [17].

The success of disease resistance breeding solely depends on the genetic variability and the evaluation tests employed for the identification of the resistant sources from the germplasm. Screening of germplasm under natural epiphytotic and glasshouse conditions using viruliferous whiteflies is followed to identify the source of resistance against the ChiLCV [18]. However, a clear understanding of the underlying genetics of disease resistance and its inheritance pattern helps to select appropriate breeding strategies for successful introgression of the resistance genes [19-21].

Moreover, a wide range of variability in chilli pepper has been exhibited for various economic and quality traits encouraging the breeders to exploit the variation for genetic improvement of the crop [22]. To break productivity limitations and to develop hybrids with desirable characters, the selection of the parents is one of the most critical responsibilities for plant breeders [23]. Although, the common technique for selecting the parents on the basis of mean performance does not always produce good hybrids. Therefore, parents should be selected on the basis of their combining ability potential. Moreover, the knowledge of gene action helps in the selection of appropriate breeding strategy for the genetic 
improvement of diverse quantitative traits [24]. Gene action is commonly measured in terms of components of genetic variance or combining ability effects and variances. The varieties or strains can be evaluated in several ways based on the combining ability of their parents and one of them is line $\times$ tester analysis [25, 26]. By using this analysis promising lines could be selected from the germplasm. As compared to diallel technique, this approach could evaluate a greater number of breeding lines at once. This, in turn, suggests the breeder whether to go for $\mathrm{F}_{1}$ hybrid development or selection in subsequent generations to realize homozygous promising lines [27]. The information on combining ability effects (general and specific combining ability) are useful for the precise interpretation of the genetic basis of promising traits [28-30]. Keeping in view of these facts and need, the present investigation was planned with the following three objectives:

1. To determine the parental genotypes reaction to ChiLCV via natural and artificial screening.

2. Identification of parents with ChiLCV resistance based on mean performance and general combining ability (GCA) effects.

3. To identify superior performing ChiLCV resistant hybrids based on expressed heterosis and specific combining ability (SCA) effects.

\section{Results}

\subsection{Screening of the Parental Genotypes}

Among the total 11 parents 7 were lines and 4 were tester genotypes. Out of seven lines, two were moderately resistant, and the remaining five showed a moderate susceptible reaction to ChiLCD (Table 1). The genotypes (L1 and L4) which showed Moderately susceptible reaction under field conditions were susceptible under artificial graft inoculation (Table 1). In contrast, the coefficient of infection (CI) was highest for the parental genotype L7. Whereas, the lowest values of CI were recorded for the testers $(<$ $5 \%)$ (Table 1). The disease incidence was maximum for the parents (Table 1). The percent of disease incidence (PDI) was above $69 \%$ for the lines and below 10\% for the testers (Table 1).

In order to confirm the presence in the graft inoculated plants, the DNA extracted from the top young leaves was subjected to a polymerase chain reaction (PCR) using geminivirus universal primers (AV494/AC1048) for confirmation of ChiLCV (Table 1). All seven lines showed amplification of $560 \mathrm{bp}$ DNA fragment specific to viral genome indicating the presence of the virus (Figure 1). Whereas, out of 4 testers determined symptomless genotypes under field conditions, none were completely free from ChiLCV infection (Table 1). All four genotypes showed highly resistant reaction under graft inoculation (Figure 1). In the four testers, the disease symptoms appeared 32.00 (T1), 34.33 (T2), 33.33 (T3) and 34.33 (T4) days after graft inoculation (Table 1). 


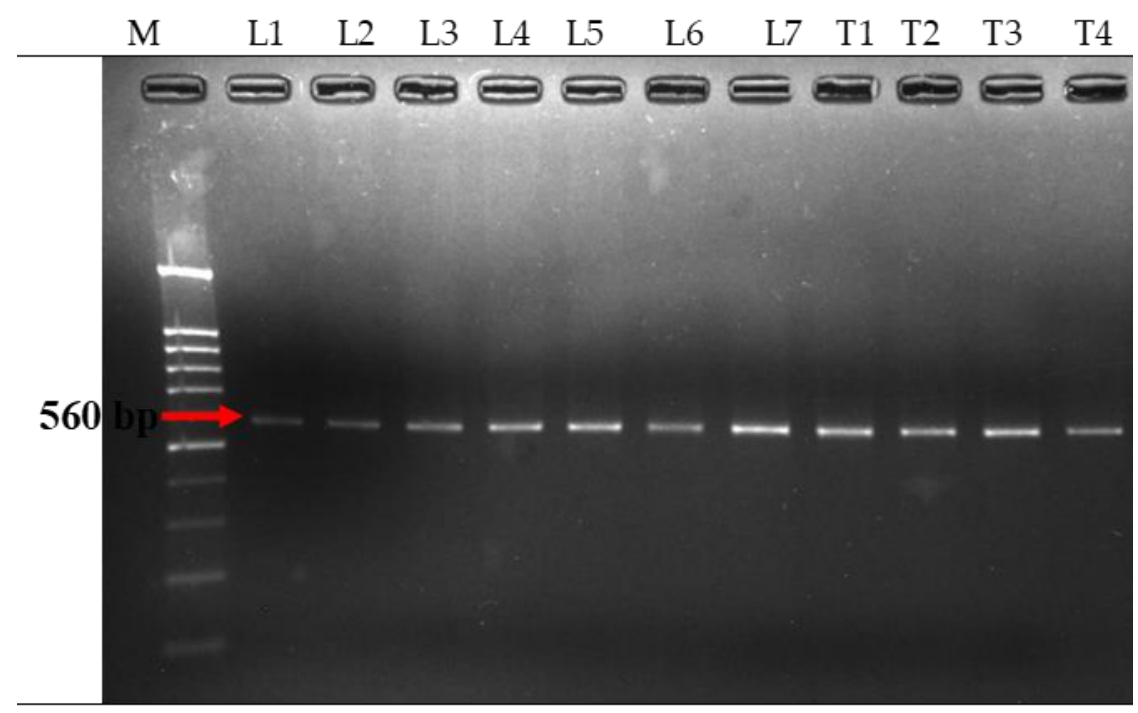

Figure 1. Virus detection in graft inoculated parents (lines and testers) as confirmed by PCR amplification of the viral genome.

Table 1. The reaction of parental genotypes (lines and testers) against ChiLCV by graft inoculation under greenhouse conditions.

\begin{tabular}{|c|c|c|c|c|c|c|c|}
\hline Genotypes & $\begin{array}{c}\text { Reaction } \\
\text { under field } \\
\text { conditions }\end{array}$ & $\begin{array}{l}\text { Appearance of } \\
\text { symptom after } \\
\text { grafting (days) }\end{array}$ & $\operatorname{PDI}^{1}(\%)$ & $\mathrm{DI}^{2}(\%)$ & $\mathrm{CI}^{3}(\%)$ & $\begin{array}{c}\text { Disease } \\
\text { reaction }^{4}\end{array}$ & $\begin{array}{c}\text { Virus } \\
\text { presence by } \\
\text { PCR }^{5}\end{array}$ \\
\hline L1 & MR & 22.33 & 69.44 & 100 & 69.44 & S & + \\
\hline L2 & MS & 21.67 & 87.22 & 100 & 87.22 & HS & + \\
\hline L3 & MS & 22.33 & 94.44 & 100 & 94.44 & HS & + \\
\hline L4 & MR & 22 & 69.44 & 100 & 69.44 & S & + \\
\hline L5 & MS & 21.67 & 94.44 & 100 & 94.44 & HS & + \\
\hline L6 & MS & 22 & 87.22 & 100 & 87.22 & HS & + \\
\hline L7 & MS & 22.67 & 100 & 100 & 100 & HS & + \\
\hline $\mathrm{T} 1$ & SL & 32 & 8.89 & 40 & 3.56 & HR & + \\
\hline $\mathrm{T} 2$ & SL & 34.33 & 8.89 & 53.33 & 4.89 & HR & + \\
\hline $\mathrm{T} 3$ & SL & 33.33 & 7.78 & 40 & 3.11 & HR & + \\
\hline $\mathrm{T} 4$ & SL & 34.33 & 7.78 & 40 & 3.11 & HR & + \\
\hline
\end{tabular}

$\mathrm{PDI}^{1}$ - Percent Disease Index, DI ${ }^{2}$ Disease Incidence, $\mathrm{CI}^{3}$ - Coefficient of Infection ${ }^{4}$ SL-Symptomless, HR-Highly resistant, RResistant, MR-Moderately resistant, MS-Moderately susceptible, S-Susceptible, HS-Highly susceptible. ${ }^{5-}$ : absence, + : presence of $550 \mathrm{bp}$ viral genome

\subsection{Field Screening of Parents and their Hybrids}

The results of field screening of parent and their hybrids are presented in Table 2. All the four testers were symptomless under field screening experiment, and among seven lines, two were moderately resistant and the remaining five were moderately susceptible (Table 2). Where, among $28 \mathrm{~F}_{1}$ hybrids, none was completely free from ChiLCV incidence (Table 2). Twelve hybrids showed moderately resistant reaction and the $\mathrm{CI}$ of disease ranged from $13.90 \%$ in the cross $\mathrm{L} 3 \times \mathrm{T} 2$ to $18.13 \%$ in the cross $\mathrm{L} 1 \times \mathrm{T} 3$ (Table 2 ). 
Eleven hybrids were moderately susceptible, among them CI ranged from $28.14 \%$ in the cross L4 $\times$ T3 to $33.88 \%$ in the cross L3 $\times$ T4 (Table 2). All of the four testers showed nil values for PDI and disease incidence (DI) (Table 2). In the case of parents, the lowest value of PDI and DI was recorded for L4 (Table 2). Among hybrid combinations L1 $\times$ T1, L3 $\times$ T2 and L6 $\times$ T3 were determined as promising with a lower value for both PDI and DI (Table 2).

Table 2. Reaction of parents and $\mathrm{F}_{1}$ hybrids to local isolate of ChiLCV under normal field conditions.

$\begin{array}{lllll}\text { Parents/Hybrids } & \mathrm{PDI}^{1} & \mathrm{DI}^{2} & \mathrm{CI}^{3} & \begin{array}{c}\text { Disease } \\ \text { reaction }\end{array}\end{array}$

\begin{tabular}{|c|c|c|c|c|}
\hline L1 & 23.06 & 81.67 & 18.85 & MR \\
\hline L2 & 36.39 & 80 & 29.53 & MS \\
\hline L3 & 37.22 & 75 & 28.39 & MS \\
\hline $\mathrm{L} 4$ & 22.5 & 75 & 16.92 & MR \\
\hline L5 & 39.44 & 86.67 & 34.42 & MS \\
\hline L6 & 38.89 & 80 & 31.32 & MS \\
\hline L7 & 38.89 & 83.33 & 32.6 & MS \\
\hline $\mathrm{T} 1$ & 0 & 0 & 0 & SL \\
\hline $\mathrm{T} 2$ & 0 & 0 & 0 & SL \\
\hline T3 & 0 & 0 & 0 & SL \\
\hline $\mathrm{T} 4$ & 0 & 0 & 0 & SL \\
\hline $\mathrm{L} 1 \times \mathrm{T} 1$ & 21.39 & 66.67 & 14.32 & MR \\
\hline $\mathrm{L} 1 \times \mathrm{T} 2$ & 24.17 & 71.67 & 17.31 & MR \\
\hline $\mathrm{L} 1 \times \mathrm{T} 3$ & 24.72 & 73.33 & 18.13 & MR \\
\hline $\mathrm{L} 1 \times \mathrm{T} 4$ & 25.56 & 65 & 16.67 & MR \\
\hline $\mathrm{L} 2 \times \mathrm{T} 1$ & 35.83 & 81.67 & 29.43 & MS \\
\hline $\mathrm{L} 2 \times \mathrm{T} 2$ & 38.33 & 85 & 32.72 & $\mathrm{MS}$ \\
\hline $\mathrm{L} 2 \times \mathrm{T} 3$ & 37.78 & 83.33 & 31.79 & MS \\
\hline $\mathrm{L} 2 \times \mathrm{T} 4$ & 39.72 & 81.67 & 32.61 & $\mathrm{MS}$ \\
\hline $\mathrm{L} 3 \times \mathrm{T} 1$ & 37.78 & 83.33 & 31.75 & MS \\
\hline $\mathrm{L} 3 \times \mathrm{T} 2$ & 21.39 & 65 & 13.9 & MR \\
\hline $\mathrm{L} 3 \times \mathrm{T} 3$ & 36.94 & 81.67 & 30.29 & MS \\
\hline $\mathrm{L} 3 \times \mathrm{T} 4$ & 39.72 & 85 & 33.88 & $\mathrm{MS}$ \\
\hline $\mathrm{L} 4 \times \mathrm{T} 1$ & 21.39 & 66.67 & 14.39 & $\mathrm{MR}$ \\
\hline $\mathrm{L} 4 \times \mathrm{T} 2$ & 25 & 65 & 16.33 & MR \\
\hline $\mathrm{L} 4 \times \mathrm{T} 3$ & 34.72 & 80 & 28.14 & MS \\
\hline $\mathrm{L} 4 \times \mathrm{T} 4$ & 35.56 & 80 & 28.78 & MS \\
\hline $\mathrm{L} 5 \times \mathrm{T} 1$ & 60.28 & 100 & 60.28 & $S$ \\
\hline $\mathrm{L} 5 \times \mathrm{T} 2$ & 59.17 & 100 & 59.17 & $S$ \\
\hline $\mathrm{L} 5 \times \mathrm{T} 3$ & 38.06 & 76.67 & 29.58 & MS \\
\hline $\mathrm{L} 5 \times \mathrm{T} 4$ & 36.67 & 78.33 & 29.25 & MS \\
\hline $\mathrm{L} 6 \times \mathrm{T} 1$ & 23.61 & 66.67 & 15.93 & $\mathrm{MR}$ \\
\hline
\end{tabular}




\begin{tabular}{ccccc}
\hline L6 $\times$ T2 & 55 & 95 & 52.61 & S \\
\hline L6 $\times$ T3 & 21.67 & 76.67 & 16.61 & MR \\
\hline L6 $\times$ T4 & 60 & 98.33 & 59.04 & S \\
\hline L7 $\times$ T1 & 20.83 & 75 & 15.79 & MR \\
\hline L7 $\times$ T2 & 55.28 & 100 & 55.28 & S \\
\hline L7 $\times$ T3 & 23.33 & 71.67 & 16.75 & MR \\
\hline L7 $\times$ T4 & 25.56 & 63.33 & 16.21 & MR \\
\hline
\end{tabular}

$\mathrm{PDI}^{1}$ - Per cent Disease Index, $\mathrm{DI}^{2}$ - Disease Incidence, $\mathrm{CI}^{3}$ - Coefficient of Infection

${ }^{4}$ SL-Symptomless, HR-Highly resistant, R-Resistant, MR-Moderately resistant, MS-Moderately susceptible, S-Susceptible, HS-Highly susceptible.

\subsection{Analysis of Variance (ANOVA) for Experimental Design}

The mean squares (MS) due to genotypes were highly significant $(\mathrm{P} \leq 0.01)$ for all the traits studied (Table 3 ). The results further indicated that the MS due to replications were significant for fruit length, fruits plant ${ }^{-1}$ and fruit weight and non-significant for primary branches plant ${ }^{-1}$, plant height, days to the first harvest, days to first flower, fruit girth, yield plant ${ }^{-1}$, yield plot ${ }^{-1}$, vitamin C, carotenoids and coefficient of infection (Table 3).

Table 3. Analysis of variance for thirteen characters in 39 treatments ( 11 parents and $28 \mathrm{~F}_{1}$ hybrids).

\begin{tabular}{|c|c|c|c|}
\hline Source of variation & Replication & Genotypes & Error \\
\hline $\mathrm{df}$ & 2 & 38 & 76 \\
\hline Plant height $(\mathrm{cm})$ & 5.99 & $174.98^{* *}$ & 2.65 \\
\hline Primary branches plant ${ }^{-1}$ & 0.28 & $1.80^{* *}$ & 0.23 \\
\hline Days to first flower & 0.19 & $29.50^{* *}$ & 0.60 \\
\hline Days to first harvest & 1.19 & $33.75^{* *}$ & 1.54 \\
\hline Fruit length $(\mathrm{cm})$ & $0.83^{* *}$ & $7.20^{* *}$ & 0.05 \\
\hline Fruit girth $(\mathrm{cm})$ & 0.03 & $0.67^{* *}$ & 0.04 \\
\hline Fruit weight (g) & $0.10^{* *}$ & $2.18^{* *}$ & 0.03 \\
\hline Fruits plant ${ }^{-1}$ & $680.67^{* *}$ & $2935.42^{* *}$ & 9.67 \\
\hline Yield plant ${ }^{-1}(\mathrm{~g})$ & 236.09 & $64451.41^{* *}$ & 403.39 \\
\hline Yield plot $^{-1}\left(\mathrm{~kg} / 6.48 \mathrm{~m}^{2}\right)$ & 0.12 & $50.42^{* *}$ & 0.13 \\
\hline Vitamin C (mg $\left.100 \mathrm{~g}^{-1}\right)$ & 4.33 & $803.89^{* *}$ & 2.39 \\
\hline Carotenoids (mg $100 \mathrm{~g}^{-1}$ ) & 0.62 & $7836.27^{* *}$ & 10.65 \\
\hline Coefficient of infection (\%) & 36.78 & $555.34^{* *}$ & 8.04 \\
\hline
\end{tabular}

Data are mean sums of squares; ${ }^{*}$ significant at $P \leq 0.05$; ${ }^{* *}$ significant at $P \leq 0.01$ 
7 of 31

\subsection{Analysis of Variance (ANOVA) for Combining Ability and its Contribution}

The results of ANOVA for combining ability for different traits are presented in Table 4. The MS due to replication were non-significant for all the studied traits except for plant height, fruit length, fruits plant ${ }^{-1}$, fruit weight and yield plant ${ }^{-1}$ (Table 4 ). Significant differences due to lines were found for all the traits (Table 4). All of the four testers were significantly different for the studied traits except for the coefficient of infection (Table 4). The hybrids differed substantially for all of traits studied. Likewise, Lines vs Testers showed significant differences for all the traits except for plant height. Similarly, the MS due to parent vs crosses showed significant differences for all the traits (Table 4). The GCA lines and SCA crosses were highly significant $(\mathrm{P} \leq 0.01)$ for all of the studied traits (Table 4). The GCA testers were observed to be significant for all the traits except for days to first harvest. The ratio of $\sigma^{2} \mathrm{GCA} / \mathrm{\sigma}^{2} \mathrm{SCA}$ was less than unity for all the characters (Table 5). The contribution of lines in the expression of traits was greater than the testers for all the characters except for the primary branches plant ${ }^{-1}$ (Table 5). 
Table 4. Analysis of variance for combining ability including parents in line $\times$ tester design.

\begin{tabular}{|c|c|c|c|c|c|c|c|c|c|c|c|c|c|c|}
\hline $\begin{array}{l}\text { Source of } \\
\text { variation }\end{array}$ & df & $\begin{array}{c}\text { Plant } \\
\text { height }(\mathrm{cm})\end{array}$ & $\begin{array}{l}\text { Primary } \\
\text { branches } \\
\text { Plant }^{-1}\end{array}$ & $\begin{array}{l}\text { Days to } \\
\text { first } \\
\text { flower }\end{array}$ & $\begin{array}{l}\text { Days to } \\
\text { first } \\
\text { harvest }\end{array}$ & $\begin{array}{l}\text { Fruit } \\
\text { length } \\
(\mathrm{cm})\end{array}$ & $\begin{array}{l}\text { Fruit } \\
\text { girth } \\
(\mathrm{cm})\end{array}$ & $\begin{array}{c}\text { Fruit } \\
\text { weight } \\
(\mathrm{g})\end{array}$ & $\begin{array}{l}\text { Fruits } \\
\text { plant }^{-1}\end{array}$ & $\begin{array}{c}\text { Yield plant } \\
{ }^{1}(\mathrm{~g})\end{array}$ & $\begin{array}{l}\text { Yield plot }{ }^{-1} \\
\left(\mathrm{~kg} / 6.48 \mathrm{~m}^{2}\right)\end{array}$ & $\begin{array}{l}\text { Vitamin } \\
\text { C (mg } \\
\left.100 \mathrm{~g}^{-1}\right)\end{array}$ & 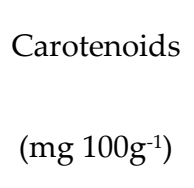 & $\begin{array}{l}\text { Coefficient } \\
\text { of infection }\end{array}$ \\
\hline Replication & 2 & $5.99^{*}$ & 0.28 & 0.19 & 1.19 & $0.83^{* *}$ & 0.03 & $0.10^{* *}$ & $680.67^{* *}$ & $236.09^{* *}$ & 0.12 & 4.33 & 0.62 & 36.78 \\
\hline Parents & 10 & $70.71^{* *}$ & $1.47^{* *}$ & $31.46^{* *}$ & $37.69^{* *}$ & $5.86^{* *}$ & $0.59^{* *}$ & $3.69^{* *}$ & $2981.09^{* *}$ & $44767.77^{* *}$ & $35.25^{* *}$ & $803.89 * *$ & $5396.66^{* *}$ & $525.34^{* *}$ \\
\hline Lines (L) & 6 & $75.35^{* *}$ & $1.42^{* *}$ & $37.08^{* *}$ & $37.23^{* *}$ & $6.58^{* *}$ & $0.72^{* *}$ & $4.87^{* *}$ & $2806.85^{* *}$ & $6499.92^{* *}$ & $5.10^{* *}$ & $569.74^{* *}$ & $1982.30^{* *}$ & $133.73^{* *}$ \\
\hline Testers $(\mathrm{T})$ & 3 & $84.98^{* *}$ & $1.76^{* *}$ & $5.17^{* *}$ & $8.73^{* *}$ & $4.22^{* *}$ & $0.51^{* *}$ & $0.86^{* *}$ & $218.97^{* *}$ & $3987.29^{* *}$ & $2.90^{* *}$ & $181.00^{* *}$ & $5341.44^{* *}$ & 0.00 \\
\hline Crosses & 27 & $157.64^{* *}$ & $1.77^{* *}$ & $26.91^{* *}$ & $25.18^{* *}$ & $5.51^{* *}$ & $0.51^{* *}$ & $1.64^{* *}$ & $2897.95^{* *}$ & $66652.64^{* *}$ & $52.06^{* *}$ & $334.30^{* *}$ & $6688.72^{* *}$ & $521.73^{* *}$ \\
\hline $\begin{array}{c}\text { Lines vs } \\
\text { Testers } \\
\end{array}$ & 1 & 0.08 & $0.87^{* *}$ & $76.62^{* *}$ & $127.37^{* *}$ & $6.40^{* *}$ & $0.08^{*}$ & $5.10^{* *}$ & $12312.91^{* *}$ & $396716.32^{* *}$ & $313.23^{* *}$ & $3608.52^{* *}$ & $26048.52^{* *}$ & $4451.08^{* *}$ \\
\hline $\begin{array}{c}\text { Parent vs } \\
\text { Crosses }\end{array}$ & 1 & $1685.85^{* *}$ & $6.07^{* *}$ & $80.04^{* *}$ & $225.74^{* *}$ & $66.42^{* *}$ & $5.65^{* *}$ & $1.59^{* *}$ & $3490.29^{* *}$ & $201854.61^{* *}$ & $157.88^{* *}$ & $1985.64^{* *}$ & $63216.15^{* *}$ & $1762.06^{* *}$ \\
\hline GCA lines & 6 & $488.43^{* *}$ & $1.05^{* *}$ & $63.74^{* *}$ & $62.80^{* *}$ & $15.08^{* *}$ & $0.99^{* *}$ & $3.05^{* *}$ & $5319.07^{* *}$ & $127365.64^{* *}$ & $99.53^{* *}$ & $2259.90^{* *}$ & $24191.51^{* *}$ & $793.80^{* *}$ \\
\hline GCA testers & 3 & $49.96^{* *}$ & $3.76^{* *}$ & $8.07^{* *}$ & 1.33 & $4.44^{* *}$ & $0.76^{* *}$ & $2.59^{* *}$ & $5173.94^{* *}$ & $127623.57^{* *}$ & $100.12^{* *}$ & $1749.66^{* *}$ & $505.09^{* *}$ & $415.26^{* *}$ \\
\hline SCA crosses & 18 & $65.32^{* *}$ & $1.68^{* *}$ & $17.77^{* *}$ & $16.62^{* *}$ & $2.50^{* *}$ & $0.30^{* *}$ & $1.01^{* *}$ & $1711.57^{* *}$ & $36253.05^{* *}$ & $28.23^{* *}$ & $225.36^{* *}$ & $1885.07^{* *}$ & $448.79^{* *}$ \\
\hline Error & 76 & 2.65 & 0.26 & 0.60 & 1.54 & 0.05 & 0.04 & 0.03 & 9.67 & 403.39 & 0.13 & 2.39 & 10.65 & 8.04 \\
\hline
\end{tabular}

Data's are mean sums of squares; ${ }^{*}$ significant at $P \leq 0.05 ;{ }^{* *}$ significant at $P \leq 0.01$ 
9 of 31

Table 5. Components of genetic variance and proportional contributions (\%) of Line, Tester and their interactions (Hybrids).

\begin{tabular}{|c|c|c|c|c|c|c|c|c|c|c|c|c|c|}
\hline & $\begin{array}{c}\text { Plant height } \\
(\mathrm{cm})\end{array}$ & $\begin{array}{l}\text { Primary } \\
\text { branches } \\
\text { Plant }^{-1}\end{array}$ & $\begin{array}{l}\text { Days } \\
\text { to first } \\
\text { flower }\end{array}$ & $\begin{array}{l}\text { Days to } \\
\text { first } \\
\text { harvest }\end{array}$ & $\begin{array}{c}\text { Fruit } \\
\text { lengt } \\
\mathrm{h} \\
(\mathrm{cm})\end{array}$ & $\begin{array}{l}\text { Fruit } \\
\text { girth } \\
(\mathrm{cm})\end{array}$ & $\begin{array}{c}\text { Fruit } \\
\text { weight } \\
\text { (g) }\end{array}$ & $\begin{array}{l}\text { Fruits } \\
\text { plant }^{-1}\end{array}$ & $\begin{array}{c}\text { Yield } \\
\text { plant }^{-1}(\mathrm{~g})\end{array}$ & $\begin{array}{l}\text { Yield } \\
\text { plot }^{-1} \\
(\mathrm{~kg} / 6.48 \\
\left.\mathrm{m}^{2}\right)\end{array}$ & $\begin{array}{l}\text { Vitamin } \\
\text { C (mg } \\
\left.100 \mathrm{~g}^{-1}\right)\end{array}$ & 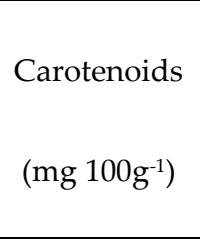 & $\begin{array}{c}\text { Coefficient of } \\
\text { infection }\end{array}$ \\
\hline \multicolumn{14}{|c|}{ Components of genetic variance } \\
\hline$\sigma^{2} g c a$ & 2.05 & 0.02 & 0.20 & 0.19 & 0.06 & 0.04 & 0.01 & 26.36 & 675.54 & 0.52 & 13.90 & 106.74 & 1.62 \\
\hline$\sigma^{2} s c a$ & 20.87 & 0.47 & 5.71 & 4.97 & 0.81 & 0.08 & 0.32 & 566.92 & 11915.02 & 9.36 & 74.34 & 623.97 & 146.85 \\
\hline$\sigma^{2} g c a / \sigma^{2} s c a$ & 0.09 & 0.04 & 0.03 & 0.03 & 0.07 & 0.5 & 0.03 & 0.04 & 0.05 & 0.05 & 0.18 & 0.17 & 0.01 \\
\hline \multicolumn{14}{|c|}{ Proportional contributions (\%) of Line, Tester and their interactions to total variance } \\
\hline Lines & 68.85 & 13.27 & 52.64 & 55.40 & 60.80 & 43.28 & 41.35 & 40.79 & 42.46 & 42.48 & 59.30 & 80.37 & 33.81 \\
\hline Testers & 3.52 & 23.54 & 3.33 & 0.59 & 8.95 & 16.66 & 17.54 & 19.84 & 21.28 & 21.39 & 22.96 & 0.84 & 8.84 \\
\hline Lines $\times$ Testers & 27.63 & 63.19 & 44.03 & 44.01 & 30.25 & 40.06 & 41.11 & 39.37 & 36.26 & 36.15 & 17.74 & 18.79 & 57.35 \\
\hline
\end{tabular}


10 of 31

\subsection{General Combining Ability Effects}

Based on general combining ability (GCA) effects, the line L1 was identified as a good general combiner for fruit weight, yield plant ${ }^{-1}$, yield plot $^{-1}$ and coefficient of infection (Table 6). L2 was identified as a good general combiner for plant height and carotenoids. Whereas, L3 for fruits plant ${ }^{-1}$, yield plant ${ }^{-1}$, yield plot $^{-1}$ and vitamin C (Table 6). In the case of L4, it showed the high GCA effects for days to first flower and harvest, fruit length, fruit girth, carotenoids, vitamin $C$ and coefficient of infection (Table 6). For L5 the high GCA effects were determined for days to first flower and harvest, fruit length, fruit girth and fruit weight; L6 for plant height, fruit girth and fruits plant ${ }^{-1}$ (Table 6). The genotype L7 for plant height, fruits plant ${ }^{-1}$, yield plant ${ }^{-1}$, yield plot $^{-1}$, vitamin $\mathrm{C}$ and coefficient of infection. Among four testers, $\mathrm{T} 1$ was identified as a good general combiner for plant height, primary branches plant ${ }^{-1}$, days to first flower, fruits plant ${ }^{-1}$, yield plant $^{-1}$, yield plot $^{-1}$, carotenoids, vitamin $C$ and coefficient of infection (Table 6). The genotype T2 for fruit length, fruit weight and vitamin C; T3 for fruit girth, vitamin C and coefficient of infection (Table 6). 
11 of 31

Table 6. Estimates of the general combining ability (GCA) effects of the parents ( $n=10)$ for the descriptors studied under the influence of chilli leaf curl virus disease.

\begin{tabular}{|c|c|c|c|c|c|c|c|c|c|c|c|c|c|}
\hline Source & $\begin{array}{l}\text { Plant } \\
\text { height } \\
(\mathrm{cm})\end{array}$ & $\begin{array}{l}\text { Primary } \\
\text { branches } \\
\text { Plant }^{-1}\end{array}$ & $\begin{array}{l}\text { Days to } \\
\text { first } \\
\text { flower }\end{array}$ & $\begin{array}{c}\text { Days to first } \\
\text { harvest }\end{array}$ & $\begin{array}{l}\text { Fruit } \\
\text { length } \\
(\mathrm{cm})\end{array}$ & $\begin{array}{l}\text { Fruit } \\
\text { girth } \\
(\mathrm{cm})\end{array}$ & $\begin{array}{l}\text { Fruit } \\
\text { weight } \\
\text { (g) }\end{array}$ & $\begin{array}{l}\text { Fruits } \\
\text { plant }^{-1}\end{array}$ & $\begin{array}{c}\text { Yield } \\
\text { plant }^{-1}(\mathrm{~g})\end{array}$ & $\begin{array}{l}\text { Yield plot }{ }^{-1} \\
\left(\mathrm{~kg} / 6.48 \mathrm{~m}^{2}\right. \\
)\end{array}$ & $\begin{array}{l}\text { Vitamin C } \\
(\mathrm{mg} 100 \mathrm{~g}- \\
\left.{ }^{1}\right)\end{array}$ & $\begin{array}{l}\text { Carotenoids } \\
\left(\mathrm{mg} 100 \mathrm{~g}^{-1}\right)\end{array}$ & $\begin{array}{l}\text { Coefficient } \\
\text { of infection } \\
\text { (CI) }\end{array}$ \\
\hline
\end{tabular}

Female parents (Lines)

\begin{tabular}{|c|c|c|c|c|c|c|c|c|c|c|c|c|c|}
\hline L1 & $-4.77^{* *}$ & $-0.37^{*}$ & $-0.85^{* *}$ & 0.64 & 0.05 & -0.12 & $0.72^{* *}$ & $3.98^{* *}$ & $91.23^{* *}$ & $2.56^{* *}$ & $-5.65^{* *}$ & $-7.90^{* *}$ & $-11.96^{* *}$ \\
\hline L2 & $4.31^{* *}$ & $-0.29^{*}$ & $2.50^{* *}$ & $2.68^{* *}$ & $-0.87^{* *}$ & $-0.29^{* *}$ & $-0.70^{* *}$ & $2.23^{*}$ & $-65.52^{* *}$ & $-1.84^{* *}$ & $-6.57^{* *}$ & $34.46^{* *}$ & $3.78^{* *}$ \\
\hline L3 & $-4.53^{* *}$ & $0.34^{*}$ & $-0.94^{* *}$ & $-1.07^{* *}$ & $-0.37^{* *}$ & $-0.31^{* *}$ & 0.06 & $23.64^{* *}$ & $128.41^{* *}$ & $3.57^{* *}$ & $17.76^{* *}$ & $-31.24^{* *}$ & -1.24 \\
\hline L4 & $-4.50^{* *}$ & 0.20 & $-1.90^{* *}$ & $-2.07^{* *}$ & $2.30^{* *}$ & $0.34^{* *}$ & $-0.37^{* *}$ & $-25.19^{* *}$ & $-141.85^{* *}$ & $-3.96^{* *}$ & $8.18^{* *}$ & $75.35^{* *}$ & $-5.47^{* *}$ \\
\hline L5 & $-5.30^{* *}$ & -0.26 & $-2.92^{* *}$ & $-3.31^{* *}$ & $0.38^{* *}$ & $0.24^{* *}$ & $0.55^{* *}$ & $-32.27^{* *}$ & $-101.82^{* *}$ & $-2.86^{* *}$ & -0.40 & $-60.40^{* *}$ & $12.68^{* *}$ \\
\hline L6 & $3.87^{* *}$ & 0.15 & $0.73^{* *}$ & 0.43 & $-0.99^{* *}$ & $0.32^{* *}$ & $-0.28^{* *}$ & $17.14^{* *}$ & $38.35^{* *}$ & $1.09^{* *}$ & $-23.74^{* *}$ & $-20.57^{* *}$ & $5.84^{* *}$ \\
\hline L7 & $10.91^{* *}$ & 0.24 & $3.37^{* * *}$ & $2.68^{* *}$ & $-0.50^{* *}$ & $-0.18^{* *}$ & 0.01 & $10.48^{* *}$ & $51.20^{* *}$ & $1.45^{* *}$ & $10.43^{* *}$ & 10.01 & $-3.63^{* *}$ \\
\hline
\end{tabular}

Male parents (Testers)

\begin{tabular}{|c|c|c|c|c|c|c|c|c|c|c|c|c|c|}
\hline $\mathrm{T} 1$ & $2.14^{* *}$ & $0.49^{* *}$ & $-0.83^{* *}$ & -0.14 & $-0.19^{* *}$ & $-0.09^{*}$ & 0.07 & $22.04^{* *}$ & $108.17^{* *}$ & $3.03^{* *}$ & $9.31^{* *}$ & $5.82^{* *}$ & $-2.80^{* *}$ \\
\hline $\mathrm{T} 2$ & 0.11 & -0.05 & -0.12 & -0.28 & $0.65^{* *}$ & -0.01 & $0.47^{* *}$ & $-11.58^{* *}$ & -6.14 & $-0.17^{*}$ & $2.17^{* *}$ & $-6.04^{* *}$ & $5.83^{* *}$ \\
\hline $\mathrm{T} 3$ & $-1.09^{* *}$ & 0.10 & $0.47^{* *}$ & 0.27 & -0.05 & $0.27^{* *}$ & $-0.28^{* *}$ & 0.46 & $-25.63^{* *}$ & $-0.72^{* *}$ & $1.07^{* *}$ & 1.06 & $-4.05^{* *}$ \\
\hline $\mathrm{T} 4$ & $-1.17^{* *}$ & $-0.54^{* *}$ & $0.48^{* *}$ & 0.15 & -0.41 & $-0.17^{* *}$ & $-0.26^{* *}$ & $-10.92^{* *}$ & $-73.40^{* *}$ & $-2.14^{* *}$ & $-12.55^{* *}$ & -0.85 & 1.02 \\
\hline
\end{tabular}


12 of 31

\subsection{Specific Combining Ability Effects.}

The SCA values for the cross combinations are presented in Table 7 . The promising cross combinations identified for plant height included L1 × T2 (7.50), L1 × T1 (6.52), L3 × T1 (5.32) and L2× T3 (5.26) (Table 7). The cross combinations L1 $\times$ T4 (-5.36), L3 $\times$ T2 (-3.22) and L7 $\times$ T1 (-2.11) were identified promising for days to first harvest (Table 7). The significantly positive SCA effects were determined in the cross combinations L3 $\times$ T2 (38.17), L7 × T3 (34.95) and L6 $\times$ T1 (32.38) for fruits plant ${ }^{-1}$ and in crosses L7 $\times$ T1 (1.17), L6 $\times$ T3 (1.13) and L1 $\times$ T2 (0.95) for fruit weight (Table 7). The cross combinations L3 $\times$ T2 (185.13), L5 × T3 (88.05), L2 × T4 (82.52), L1 × T1 (81.20) and L7 × T1 (73.30) were identified promising for yield plant ${ }^{-}$ 1 (Table 7). The significant cross combinations for vitamin C content were L5 × T4 (11.55), L3 × T2 (30.29) and L5 $\times$ T3 (9.26) (Table 7). In the case of coefficient of infection, the direction of selection was for lowest values the cross combinations L3 × T2 (-16.56), L6 × T1 (-14.90), L5 × T4 (-13.29), L6 × T3 (-12.86), L4 × T2 (11.51) and L7 $\times$ T4 (-10.21) were identified promising (Table 7). 
13 of 31

Table 7. Estimates of the specific combining ability (SCA) effects of the hybrids $(n=28)$ for the descriptors studied under the influence of chilli leaf curl virus disease.

\begin{tabular}{|c|c|c|c|c|c|c|c|c|c|c|c|c|c|}
\hline $\begin{array}{c}\text { Hybrid } \\
\mathrm{s}\end{array}$ & $\begin{array}{l}\text { Plant } \\
\text { height } \\
(\mathrm{cm})\end{array}$ & $\begin{array}{c}\text { Primary } \\
\text { branche } \\
\text { s plant } \\
1\end{array}$ & $\begin{array}{l}\text { Days to } \\
\text { first } \\
\text { flower }\end{array}$ & $\begin{array}{l}\text { Days to } \\
\text { first } \\
\text { harvest }\end{array}$ & $\begin{array}{c}\text { Fruit } \\
\text { length } \\
(\mathrm{cm})\end{array}$ & $\begin{array}{l}\text { Fruit } \\
\text { girth } \\
(\mathrm{cm})\end{array}$ & $\begin{array}{l}\text { Fruit } \\
\text { weight } \\
\text { (g) }\end{array}$ & $\begin{array}{l}\text { Fruits } \\
\text { plant }^{-1}\end{array}$ & $\begin{array}{c}\text { Yield } \\
\text { plant }^{-1}(\mathrm{~g})\end{array}$ & $\begin{array}{l}\text { Yield plot }{ }^{-1} \\
\left(\mathrm{~kg} / 6.48 \mathrm{~m}^{2}\right)\end{array}$ & $\begin{array}{l}\text { Vitamin C } \\
\left(\mathrm{mg} 100 \mathrm{~g}^{-1}\right)\end{array}$ & $\begin{array}{c}\text { Carotenoids } \\
\left(\mathrm{mg} 100 \mathrm{~g}^{-1}\right)\end{array}$ & $\begin{array}{l}\text { Coefficient of } \\
\text { Infection (CI) } \\
(\%)\end{array}$ \\
\hline $\mathrm{L} 1 \times \mathrm{T} 1$ & $6.25^{* *}$ & 0.12 & $1.00^{* *}$ & $3.93^{* *}$ & $-0.51^{* *}$ & 0.11 & $-0.35^{* *}$ & $17.21^{* *}$ & $81.20^{* *}$ & $2.28^{* *}$ & $7.61^{* *}$ & $10.43^{* *}$ & 1.76 \\
\hline $\mathrm{L} 1 \times \mathrm{T} 2$ & $7.50^{* *}$ & -0.13 & $3.78^{* *}$ & $2.07^{* *}$ & $1.36^{* *}$ & 0.07 & $0.95^{* *}$ & $-30.50^{* *}$ & $-95.94^{* *}$ & $-2.68^{* *}$ & $-2.25^{* *}$ & $-24.38^{* *}$ & $-4.98^{* *}$ \\
\hline $\mathrm{L} 1 \times \mathrm{T} 3$ & $-5.44^{* *}$ & -0.37 & 0.17 & -0.64 & $-0.92^{* *}$ & $-0.29^{*}$ & $-0.68^{* *}$ & $6.12^{* *}$ & $-45.36^{* *}$ & $-1.26^{* *}$ & $-7.82^{* *}$ & -0.81 & $4.81^{* *}$ \\
\hline $\mathrm{L} 1 \times \mathrm{T} 4$ & $-8.31^{* *}$ & 0.38 & $-4.95^{* *}$ & $-5.36^{* *}$ & 0.07 & 0.11 & 0.08 & $7.17^{* *}$ & $60.10^{* *}$ & $1.66^{* *}$ & $2.46^{* *}$ & $14.76^{* *}$ & -1.58 \\
\hline $\mathrm{L} 2 \times \mathrm{T} 1$ & -1.88 & -0.22 & $1.05^{*}$ & -0.11 & $0.62^{* *}$ & -0.12 & 0.04 & $10.63^{* *}$ & $37.04^{* *}$ & $1.05^{* *}$ & $7.52^{* *}$ & $-25.57^{* *}$ & 1.21 \\
\hline $\mathrm{L} 2 \times \mathrm{T} 2$ & $-2.84^{* *}$ & -0.23 & -0.03 & 1.03 & $0.72^{* *}$ & 0.09 & -0.16 & $-14.75^{* *}$ & $-68.78^{* *}$ & $-1.92^{* *}$ & $7.33^{* *}$ & $-4.38^{*}$ & $-5.46^{* *}$ \\
\hline $\mathrm{L} 2 \times \mathrm{T} 3$ & $5.26^{* *}$ & -0.01 & $-1.28^{* *}$ & -0.52 & $-0.59^{* *}$ & $0.49^{* *}$ & -0.07 & $-7.80^{* *}$ & $-50.78^{* *}$ & $-1.41^{* *}$ & $-11.57^{* *}$ & 3.19 & $4.93^{* *}$ \\
\hline $\mathrm{L} 2 \times \mathrm{T} 4$ & -0.54 & $0.46^{* *}$ & 0.27 & -0.40 & $-0.75^{* *}$ & $-0.46^{* *}$ & 0.20 & $11.92^{* *}$ & $82.52^{* *}$ & $2.28^{* *}$ & $-3.29^{* *}$ & $26.76^{* *}$ & -0.68 \\
\hline $\mathrm{L} 3 \times \mathrm{T} 1$ & $5.35^{* *}$ & 0.04 & $1.34^{* *}$ & 0.64 & $-0.28^{* *}$ & $0.43^{* *}$ & -0.09 & $-21.45^{* *}$ & $-108.31^{* *}$ & $-3.06^{* *}$ & 1.19 & $29.43^{* *}$ & $5.72^{* *}$ \\
\hline $\mathrm{L} 3 \times \mathrm{T} 2$ & $-4.15^{* *}$ & $1.08^{* *}$ & $-2.83^{* *}$ & $-3.22^{* *}$ & $-0.86^{* *}$ & -0.08 & -0.09 & $38.17^{* *}$ & $185.13^{* *}$ & $5.14^{* *}$ & $9.33^{* *}$ & $30.29 * *$ & $-16.56^{* *}$ \\
\hline $\mathrm{L} 3 \times \mathrm{T} 3$ & $-3.54^{* *}$ & -0.00 & $3.97^{* *}$ & $3.23^{* *}$ & $0.84^{* *}$ & $-0.32^{* *}$ & $0.37^{* *}$ & $-7.55^{* *}$ & 5.26 & 0.18 & $-2.90^{* *}$ & $-24.81^{* *}$ & $7.30^{* *}$ \\
\hline $\mathrm{L} 3 \times \mathrm{T} 4$ & $2.33^{*}$ & $-1.13^{* *}$ & $-2.48^{* *}$ & -0.65 & $0.29^{* *}$ & -0.03 & -0.19 & $-9.17^{* *}$ & $-82.08^{* *}$ & $-2.26^{* *}$ & $-7.62^{* *}$ & $-34.90^{* *}$ & $3.53^{*}$ \\
\hline $\mathrm{L} 4 \times \mathrm{T} 1$ & $-4.79^{* *}$ & -0.38 & -0.46 & -0.36 & 0.16 & -0.04 & 0.04 & $18.38^{* *}$ & $80.94^{* *}$ & $2.27^{* *}$ & $-2.56^{* *}$ & $15.85^{* *}$ & $-3.57^{*}$ \\
\hline $\mathrm{L} 4 \times \mathrm{T} 2$ & 0.22 & $1.29^{* *}$ & 0.63 & 0.78 & $0.35^{*}$ & 0.07 & 0.03 & $12.67^{* *}$ & $54.17^{* *}$ & $1.52^{* *}$ & $7.25^{* *}$ & $12.37^{* *}$ & $-11.51^{* *}$ \\
\hline $\mathrm{L} 4 \times \mathrm{T} 3$ & 0.32 & 0.19 & $-1.41^{* *}$ & $-1.77^{*}$ & $-0.72^{* *}$ & 0.03 & -0.14 & $-20.71^{* *}$ & $-98.49^{* *}$ & $-2.76^{* *}$ & $2.68^{* *}$ & $-11.06^{* *}$ & $9.77^{* *}$ \\
\hline $\mathrm{L} 4 \times \mathrm{T} 4$ & $4.25^{* *}$ & $-1.09^{* *}$ & $1.24^{* *}$ & 1.35 & 0.21 & -0.06 & 0.07 & $-10.33^{* *}$ & $-36.62^{* *}$ & $-1.03^{* *}$ & $-7.37^{* *}$ & $-17.15^{* *}$ & $5.31^{* *}$ \\
\hline $\mathrm{L} 5 \times \mathrm{T} 1$ & -0.10 & -0.07 & -0.25 & -1.11 & $-0.89^{* *}$ & 0.01 & $-0.28^{*}$ & $-39.20^{* *}$ & $-221.72^{* *}$ & $-6.19^{* *}$ & $-12.98^{* *}$ & $-7.74^{* *}$ & $15.58^{* *}$ \\
\hline $\mathrm{L} 5 \times \mathrm{T} 2$ & -1.14 & $-0.69^{*}$ & 0.07 & 0.03 & 0.20 & $-0.61^{* *}$ & 0.01 & $11.42^{* *}$ & $53.04^{* *}$ & $1.51^{* *}$ & $-7.83^{* *}$ & $14.45^{* *}$ & $6.66^{* *}$ \\
\hline $\mathrm{L} 5 \times \mathrm{T} 3$ & -1.11 & 0.28 & -0.06 & 0.48 & 0.23 & 0.18 & 0.08 & $13.04^{* *}$ & $88.05^{* *}$ & $2.40^{* *}$ & $9.26^{* *}$ & $-11.98^{* *}$ & $-8.95^{* *}$ \\
\hline $\mathrm{L} 5 \times \mathrm{T} 4$ & $2.35^{*}$ & 0.48 & 0.24 & 0.60 & $0.46^{* *}$ & $0.42^{* *}$ & 0.19 & $14.75^{* *}$ & $80.63^{* *}$ & $2.28^{* *}$ & $11.55^{* *}$ & $5.26^{*}$ & $-13.29^{* *}$ \\
\hline $\mathrm{L} 6 \times \mathrm{T} 1$ & -1.45 & $0.71^{*}$ & $-0.95^{*}$ & -0.86 & -0.19 & 0.02 & $-0.53^{* *}$ & $32.38^{* *}$ & $57.55^{* *}$ & $1.61^{* *}$ & $-5.98^{* *}$ & $-27.24^{* *}$ & $-14.90^{* *}$ \\
\hline $\mathrm{L} 6 \times \mathrm{T} 2$ & -0.23 & $-1.43^{* *}$ & $-2.22^{* *}$ & $-1.72^{*}$ & $-1.63^{* *}$ & -0.01 & $-0.74^{* *}$ & $-13.00^{* *}$ & $-119.65^{* *}$ & $-3.35^{* *}$ & $-5.50^{* *}$ & 1.29 & $10.83^{* *}$ \\
\hline
\end{tabular}


14 of 31

\begin{tabular}{|c|c|c|c|c|c|c|c|c|c|c|c|c|c|}
\hline $\mathrm{L} 6 \times \mathrm{T} 3$ & -0.31 & 0.28 & $-0.58^{* *}$ & -0.27 & $1.03^{* *}$ & -0.02 & $1.13^{* *}$ & $-18.05^{* *}$ & $53.72^{* *}$ & $1.51^{* *}$ & $7.26^{* *}$ & $41.86^{* *}$ & $-12.86^{* *}$ \\
\hline $\mathrm{L} 6 \times \mathrm{T} 4$ & $1.99^{*}$ & 0.44 & $3.75^{* *}$ & $2.85^{* *}$ & $0.79^{* *}$ & 0.01 & 0.15 & -1.33 & 8.38 & 0.24 & $4.21^{* *}$ & $-15.90^{* *}$ & $16.93^{* *}$ \\
\hline $\mathrm{L} 7 \times \mathrm{T} 1$ & $-3.37^{* *}$ & -0.20 & $-1.73^{* *}$ & $-2.11^{* *}$ & $1.09^{* *}$ & $-0.41^{* *}$ & $1.17^{* *}$ & $-17.95^{* *}$ & $73.30^{* *}$ & $2.05^{* *}$ & $5.19^{* *}$ & $4.85^{*}$ & $-5.80^{* *}$ \\
\hline $\mathrm{L} 7 \times \mathrm{T} 2$ & 0.64 & 0.11 & 0.60 & 1.03 & -0.13 & $0.46^{* *}$ & 0.00 & $-4.00^{*}$ & -7.98 & -0.22 & $-8.33^{* *}$ & $-29.63^{* *}$ & $21.02^{* *}$ \\
\hline $\mathrm{L} 7 \times \mathrm{T} 3$ & $4.81^{* *}$ & -0.37 & -0.81 & -0.52 & 0.13 & -0.06 & $-0.68^{* *}$ & $34.95^{* *}$ & $47.60^{* *}$ & $1.33^{* *}$ & $3.10^{* *}$ & 3.61 & $-5.00^{* *}$ \\
\hline $\mathrm{L} 7 \times \mathrm{T} 4$ & $-2.08^{*}$ & 0.46 & $1.94^{* *}$ & $1.60^{*}$ & $-1.09^{* *}$ & 0.01 & $-0.49^{* *}$ & $-13.00^{* *}$ & $-112.93^{* *}$ & $-3.16^{* *}$ & 0.05 & $21.18^{* *}$ & $-10.21^{* *}$ \\
\hline
\end{tabular}

$* * * *$ significant at $P \leq 0.05$ and $P \leq 0.01$, respectively 


\subsection{Better Parent (BPH) and Midparent Heterosis (MPH)}

Out of 28 F1 hybrids, seven hybrids exhibited high heterobeltiosis for yield plant ${ }^{-1}$. The hybrid combinations L3 × T2 (55.87\%), L7 × T1 (50.46\%), and L1 × T1 $(41.78 \%)$, showed the significant BPH for the yield plant ${ }^{-1}$ (Table 8). All of these hybrids showed a significant and a positive SCA effects along with high per se performance suggested the importance of non-additive gene action (Table 7). In this direction, the top hybrid combination for yield plant ${ }^{-1}(\mathrm{~L} 3 \times \mathrm{T} 2)$ also showed substantial and desirable heterobeltiosis for primary branches, days to flower, days to harvest, fruit length, fruits plant ${ }^{-1}$, yield plot $^{-1}$, vitamin C, carotenoids and coefficient of infection (Table 8). Whereas, the second beset hybrid, for yield plant ${ }^{-1}(\mathrm{~L} 7 \times \mathrm{T} 1)$ showed significant and desirable heterobeltiosis for plant height, days to first flower, days to first harvest, fruit length, fruits plant ${ }^{-1}$, fruit weight, yield plot $^{-1}$, vitamin C, carotenoids and coefficient of infection (Table 8).

The cross combination L6 $\times$ T1 also showed significant and desirable heterobeltiosis for plant height, primary branches plant ${ }^{-1}$, days to first flower, days to first harvest, fruit length, fruit girth, fruits plant1 , yield plot ${ }^{-1}$, carotenoids and coefficient of infection (Table 8). The range of significant negative heterobeltiosis for coefficient of infection varied from $-24.89 \%$ in the cross L $5 \times \mathrm{T} 4$ to $-61.36 \%$ in the cross L7 $\times$ T4. Out of 28 evaluated crosses, nine showed significant negative BPH for coefficient of infection (Table 8). Top hybrids L7 × T4 (-61.36\%), L7 × T3 (-60.88\%), L7 × T1 (-59.35\%) and L6 × T1 ($56.52 \%$ ) exhibited highly negatively significant BPH for coefficient of infection (Table 8 ).

The results of MPH are presented in Table 9. In case of Primary branches plant ${ }^{-1}$ the hybrids which showed high significant MPH heterosis values were L4 × T2 $(103.40 \%), \mathrm{L} 3 \times \mathrm{T} 2(72.86 \%)$ and L7 $\times$ T2 $(42.85 \%)$ (Table 9). Whereas, for fruit length, the hybrid combination L4 $\times$ T4 exhibited highest significant positive MPH of $87.44 \%$ followed by L1 $\times \mathrm{T} 2(81.22 \%), \mathrm{L} 4 \times \mathrm{T} 1(75.90 \%), \mathrm{L} 4 \times \mathrm{T} 2(75.28 \%)$ and L1 $\times$ T4 (68.93\%) (Table 9). The hybrid L1 $\times$ T2 exhibited the highest significant positive MPH of $65.27 \%$, followed by L1 $\times$ T4 $(44.22 \%)$ for fruit weight (Table 9). The crosses L3 $\times$ T2 $(100.17 \%), \mathrm{L} 7 \times$ T1 $(91.59 \%)$ and L1 $\times$ T1 (88.21\%) were determined with significant MPH values for Yield Plot ${ }^{-1}($ Table 9). None of the hybrids displayed a significant negative MPH for the coefficient of infection (Table 9). 
Table 8. Heterosis over better parent values $(\mathrm{BPH}, \%)$ intended for thirteen traits in 28 hybrids of chilli pepper produced in a $\mathrm{L} \times \mathrm{T}$ matting design.

\begin{tabular}{|c|c|c|c|c|c|c|c|c|c|c|c|c|c|}
\hline \multirow[b]{2}{*}{ Hybrids } & \multirow[b]{2}{*}{$\begin{array}{l}\text { Plant } \\
\text { height } \\
(\mathrm{cm})\end{array}$} & \multirow[b]{2}{*}{$\begin{array}{c}\text { Primary } \\
\text { branches } \\
\text { plant }^{-1}\end{array}$} & \multirow[b]{2}{*}{$\begin{array}{l}\text { Days to } \\
\text { first } \\
\text { flower }\end{array}$} & \multirow[b]{2}{*}{$\begin{array}{l}\text { Days to } \\
\text { first } \\
\text { harvest }\end{array}$} & \multirow[b]{2}{*}{$\begin{array}{l}\text { Fruit } \\
\text { length } \\
(\mathrm{cm})\end{array}$} & \multirow[b]{2}{*}{$\begin{array}{l}\text { Fruit } \\
\text { girth } \\
(\mathrm{cm})\end{array}$} & \multirow[b]{2}{*}{$\begin{array}{c}\text { Fruit } \\
\text { weight } \\
(\mathrm{g})\end{array}$} & \multirow[b]{2}{*}{$\begin{array}{l}\text { Fruits } \\
\text { plant }^{-1}\end{array}$} & \multirow[b]{2}{*}{$\begin{array}{l}\text { Yield } \\
\text { plant }^{-1} \\
(\mathrm{~g})\end{array}$} & \multicolumn{3}{|c|}{ Vitamin } & \multirow{2}{*}{$\begin{array}{c}\text { Coefficient } \\
\text { of } \\
\text { infection } \\
(\mathrm{CI})(\%) \\
\end{array}$} \\
\hline & & & & & & & & & & $\begin{array}{l}\text { Yield plot }{ }^{-1} \\
\left(\mathrm{~kg} / 6.48 \mathrm{~m}^{2}\right)\end{array}$ & $\begin{array}{c}\mathrm{C} \\
(\mathrm{mg} 100 \\
\left.\mathrm{g}^{-1}\right)\end{array}$ & $\begin{array}{l}\text { Carotenoids } \\
\left(\mathrm{mg} 100 \mathrm{~g}^{-1}\right)\end{array}$ & \\
\hline $\mathrm{L} 1 \times \mathrm{T} 1$ & $8.45^{* *}$ & -2.62 & $-15.12^{* *}$ & -3.51 & $40.22^{* *}$ & $20.57^{* *}$ & $36.84^{* *}$ & $4.09^{*}$ & $41.78^{* *}$ & $42.31^{* *}$ & $12.62^{* *}$ & $28.30^{* *}$ & $-30.25^{*}$ \\
\hline $\mathrm{L} 1 \times \mathrm{T} 2$ & $31.45^{* *}$ & 30 & -1.46 & $-8.62^{* *}$ & $66.16^{* *}$ & -1.15 & $51.65^{* *}$ & $-48.49^{* *}$ & $-8.44^{* *}$ & $-8.55^{* *}$ & $-3.88^{* *}$ & $6.53^{* *}$ & -18.25 \\
\hline $\mathrm{L} 1 \times \mathrm{T} 3$ & 4.31 & $-21.35^{*}$ & $-7.44^{* *}$ & $-5.86^{* *}$ & 1.37 & $13.13^{*}$ & 4.63 & $-17.03^{* *}$ & -3.09 & -3.13 & $-10.36^{* *}$ & $20.84^{* *}$ & -18.78 \\
\hline $\mathrm{L} 1 \times \mathrm{T} 4$ & $-12.92^{* *}$ & -3.12 & $-28.89^{* *}$ & $-17.86^{* *}$ & $48.12^{* *}$ & $15.68^{* *}$ & $39.47^{* *}$ & $-23.71^{* *}$ & $6.34^{*}$ & $6.19^{* *}$ & $-13.59^{* *}$ & $22.46^{* *}$ & -27.25 \\
\hline $\mathrm{L} 2 \times \mathrm{T} 1$ & $10.19^{* *}$ & -8.68 & $-5.61^{* *}$ & $-7.02^{* *}$ & $11.61^{* *}$ & $19.84^{* *}$ & 7.3 & $11.98^{* *}$ & $21.73^{* *}$ & $22.04^{* *}$ & $9.18^{* *}$ & $3.55^{* *}$ & 11.8 \\
\hline $\mathrm{L} 2 \times \mathrm{T} 2$ & $11.40^{* *}$ & 29.13 & $-5.88^{* *}$ & $-6.90^{* *}$ & $27.23^{* *}$ & -5.17 & -4.03 & $-31.30^{* *}$ & $-21.36^{* *}$ & $-21.67^{* *}$ & 2.22 & $6.99^{* *}$ & $20.17^{*}$ \\
\hline $\mathrm{L} 2 \times \mathrm{T} 3$ & $24.74^{* *}$ & -10.81 & $-7.72^{* *}$ & $-7.02^{* *}$ & $-8.20^{* *}$ & $32.66^{* *}$ & $-14.29^{* *}$ & $-17.36^{* *}$ & $-21.66^{* *}$ & $-21.96^{* *}$ & $-16.77^{* *}$ & $12.38^{* *}$ & $22.36^{*}$ \\
\hline $\mathrm{L} 2 \times \mathrm{T} 4$ & $13.38^{* *}$ & 1.71 & $-5.15^{* *}$ & $-7.02^{* *}$ & $-15.18^{* *}$ & -9.06 & 3 & $-11.25^{* *}$ & -5.5 & $-5.92^{* *}$ & $-21.84^{* *}$ & $20.34^{* *}$ & $20.05^{*}$ \\
\hline $\mathrm{L} 3 \times \mathrm{T} 1$ & $7.27^{* *}$ & 12.42 & $-16.77^{* *}$ & $-12.28^{* *}$ & $7.49^{*}$ & $24.47^{* *}$ & $6.67^{*}$ & $16.08^{* *}$ & $23.00^{* *}$ & $22.84^{* *}$ & $15.99^{* *}$ & $18.89^{* *}$ & -0.48 \\
\hline $\mathrm{L} 3 \times \mathrm{T} 2$ & $6.00^{*}$ & $49.18^{* *}$ & $-26.18^{* *}$ & $-20.69^{* *}$ & $12.06^{* *}$ & $-10.63^{*}$ & $14.29^{* *}$ & $37.33^{* *}$ & $55.87^{* *}$ & $56.04^{* *}$ & $16.86^{* *}$ & $14.06^{* *}$ & $-53.42^{* *}$ \\
\hline $\mathrm{L} 3 \times \mathrm{T} 3$ & $9.29^{* *}$ & 4.86 & $-6.05^{* *}$ & $-5.36^{* *}$ & $23.50^{* *}$ & 5.72 & $8.89^{* *}$ & $9.81^{* *}$ & $19.28^{* *}$ & $19.54^{* *}$ & $5.23^{* *}$ & $-7.03^{* *}$ & 0.81 \\
\hline $\mathrm{L} 3 \times \mathrm{T} 4$ & $9.75^{* *}$ & $-27.40^{*}$ & $-23.59^{* *}$ & $-12.50^{* *}$ & $13.49^{* *}$ & 4.53 & -2.96 & -0.82 & $-6.06^{*}$ & $-6.14^{* *}$ & $-10.76^{* *}$ & $-12.30^{* *}$ & 5.82 \\
\hline $\mathrm{L} 4 \times \mathrm{T} 1$ & $-11.11^{* *}$ & -0.97 & $-22.13^{* *}$ & $-15.79^{* *}$ & $47.51^{* *}$ & $23.83^{* *}$ & -1.82 & 2.84 & 0.89 & 0.89 & $20.47^{* *}$ & $38.28^{* *}$ & -17.86 \\
\hline $\mathrm{L} 4 \times \mathrm{T} 2$ & $15.80^{* *}$ & $99.17^{* *}$ & $-13.68^{* *}$ & $-15.52^{* *}$ & $63.78^{* *}$ & $11.49^{*}$ & $6.55^{*}$ & $-27.58^{* *}$ & $-23.26^{* *}$ & $-23.55^{* *}$ & $23.15^{* *}$ & $32.45^{* *}$ & -13.16 \\
\hline $\mathrm{L} 4 \times \mathrm{T} 3$ & $13.42^{* *}$ & 5.97 & $-15.33^{* *}$ & $-12.96^{* *}$ & $35.96^{* *}$ & $37.58^{* *}$ & $-13.45^{* *}$ & $-44.07^{* *}$ & $-52.73^{* *}$ & $-53.39^{* *}$ & $17.45^{* *}$ & $26.24^{* *}$ & $64.00^{* *}$ \\
\hline $\mathrm{L} 4 \times \mathrm{T} 4$ & $13.81^{* *}$ & $-29.19^{*}$ & $-14.64^{* *}$ & $-10.71^{* *}$ & $44.88^{* *}$ & $20.47^{* *}$ & $-8.36^{*}$ & $-44.85^{* *}$ & $-50.83^{* *}$ & $-51.46^{* *}$ & $-6.38^{* *}$ & $23.19^{* *}$ & $68.14^{* *}$ \\
\hline $\mathrm{L} 5 \times \mathrm{T} 1$ & -4.05 & -4.53 & $-24.40^{* *}$ & $-19.30^{* *}$ & $-24.11^{* *}$ & -7.61 & $-31.54^{* *}$ & $-23.79^{* *}$ & $-27.24^{* *}$ & $-27.68^{* *}$ & $6.71^{* *}$ & $-7.54^{* *}$ & $52.27^{* *}$ \\
\hline $\mathrm{L} 5 \times \mathrm{T} 2$ & 3.99 & $-24.30^{*}$ & $-18.27^{* *}$ & $-18.97^{* *}$ & -1.19 & $-20.56^{* *}$ & $-22.42^{* *}$ & $23.08^{* *}$ & $8.50^{*}$ & $8.64^{* *}$ & $4.59^{* *}$ & $-2.87^{*}$ & $51.41^{* *}$ \\
\hline $\mathrm{L} 5 \times \mathrm{T} 3$ & 1.53 & -2.73 & $-14.35^{* *}$ & $-11.11^{* *}$ & $-9.09^{* *}$ & 5.08 & $-31.54^{* *}$ & $19.28^{* *}$ & $11.95^{* *}$ & $11.49^{* *}$ & $21.55^{* *}$ & $-11.61^{* * *}$ & $-27.12^{* *}$ \\
\hline $\mathrm{L} 5 \times \mathrm{T} 4$ & $8.17^{* *}$ & -6.43 & $-20.24^{* *}$ & $-14.29^{* *}$ & $-10.67^{* *}$ & 0.25 & $-29.71^{* *}$ & $14.04^{* *}$ & -1.01 & -1.02 & $9.54^{* *}$ & $-5.39 * *$ & $-24.89^{* *}$ \\
\hline
\end{tabular}




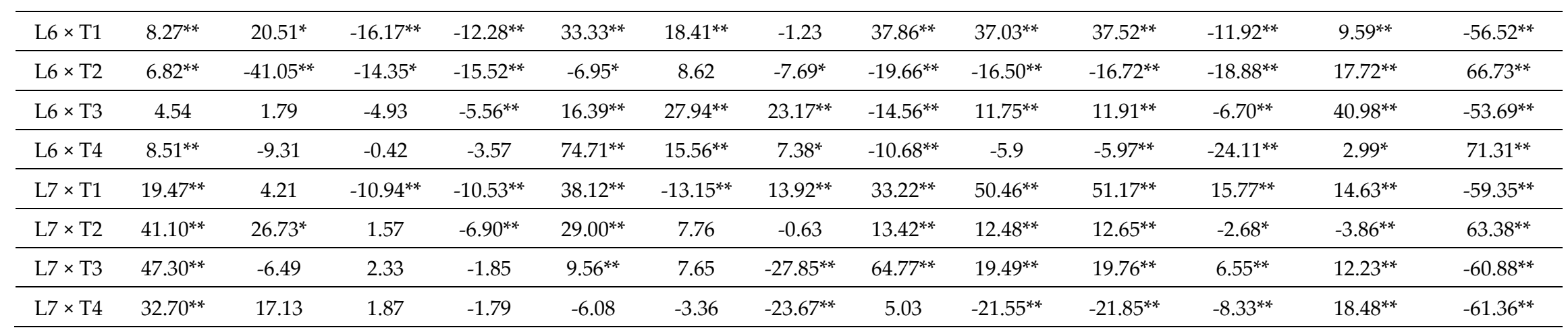

***: Significant at $P \leq 0.05$ and $P \leq 0.01$, respectively

Table 9. Heterosis over midparent values ( $\mathrm{MPH}, \%)$ intended for thirteen traits in 28 hybrids of chilli pepper produced in a $\mathrm{L} \times \mathrm{T}$ matting design.

\begin{tabular}{|c|c|c|c|c|c|c|c|c|c|c|c|c|c|}
\hline Hybrids & $\begin{array}{l}\text { Plant } \\
\text { height } \\
(\mathrm{cm})\end{array}$ & $\begin{array}{l}\text { Primary } \\
\text { branches } \\
\text { plant }^{-1}\end{array}$ & $\begin{array}{l}\text { Days to } \\
\text { first } \\
\text { flower }\end{array}$ & $\begin{array}{l}\text { Days to } \\
\text { first } \\
\text { harvest }\end{array}$ & $\begin{array}{l}\text { Fruit } \\
\text { length } \\
(\mathrm{cm})\end{array}$ & $\begin{array}{l}\text { Fruit } \\
\text { girth } \\
(\mathrm{cm})\end{array}$ & $\begin{array}{c}\text { Fruit } \\
\text { weight } \\
(\mathrm{g})\end{array}$ & $\begin{array}{l}\text { Fruits } \\
\text { plant }^{-1}\end{array}$ & $\begin{array}{l}\text { Yield } \\
\text { plant }^{-1} \\
(\mathrm{~g})\end{array}$ & $\begin{array}{l}\text { Yield plot }{ }^{-1} \\
\left(\mathrm{~kg} / 6.48 \mathrm{~m}^{2}\right)\end{array}$ & $\begin{array}{l}\text { Vitamin } \\
\text { C } \\
(\mathrm{mg} 100 \\
\left.\mathrm{g}^{-1}\right)\end{array}$ & 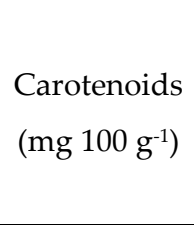 & $\begin{array}{l}\text { Coefficient } \\
\text { of } \\
\text { infection } \\
\text { (CI) }(\%)\end{array}$ \\
\hline $\mathrm{L} 1 \times \mathrm{T} 1$ & $23.02^{* *}$ & $21.32^{*}$ & $-8.18^{* *}$ & 1.85 & $44.94^{* *}$ & $27.34^{* *}$ & $42.79^{* *}$ & $31.79^{* *}$ & $86.78^{* *}$ & $88.21^{* *}$ & $33.33^{* *}$ & $48.92^{* *}$ & 39.51 \\
\hline $\mathrm{L} 1 \times \mathrm{T} 2$ & $35.71^{* *}$ & $30.00^{*}$ & $4.61^{* *}$ & -2.75 & $81.22^{* *}$ & $9.21^{*}$ & $65.27^{* *}$ & $-28.87^{* *}$ & $19.63^{* *}$ & $19.95^{* *}$ & $11.24^{* *}$ & $32.24^{* *}$ & $63.49^{*}$ \\
\hline $\mathrm{L} 1 \times \mathrm{T} 3$ & $5.45^{*}$ & -3 & -3.17 & -3.17 & $15.58^{* *}$ & $16.06^{* *}$ & $11.29^{* *}$ & $7.99^{* *}$ & $20.94^{* *}$ & $21.70^{* *}$ & $-2.98^{*}$ & $24.22^{* *}$ & $62.43^{*}$ \\
\hline $\mathrm{L} 1 \times \mathrm{T} 4$ & $-7.09^{* *}$ & 11.34 & $-22.70^{* *}$ & $-14.02^{* *}$ & $68.93^{* *}$ & $16.70^{* *}$ & $44.22^{* *}$ & 1.29 & $46.75^{* *}$ & $47.25^{* *}$ & $-9.49^{* *}$ & $24.79^{* *}$ & 45.5 \\
\hline $\mathrm{L} 2 \times \mathrm{T} 1$ & $13.57^{* *}$ & 13.77 & $-5.22^{* *}$ & $-7.02^{* *}$ & $29.79^{* *}$ & $21.53^{* *}$ & $13.12^{* *}$ & $35.10^{* *}$ & $53.25^{* *}$ & $54.20^{* *}$ & $30.43^{* *}$ & $31.93^{* *}$ & $123.61^{* *}$ \\
\hline $\mathrm{L} 2 \times \mathrm{T} 2$ & $19.41^{* *}$ & $29.13^{*}$ & $-4.36^{* *}$ & $-6.09^{* *}$ & $32.29^{* *}$ & $11.30^{*}$ & 3.56 & $-8.91^{* *}$ & -1.88 & -1.91 & $19.41^{* *}$ & $44.42^{* *}$ & $140.33^{* *}$ \\
\hline $\mathrm{L} 2 \times \mathrm{T} 3$ & $36.36^{* *}$ & 10 & $-4.80^{* *}$ & $-4.50^{* *}$ & $-7.23^{* *}$ & $45.39^{* *}$ & $-9.76^{* *}$ & 2.74 & $-6.99^{*}$ & $-6.75^{* *}$ & $-9.00^{* *}$ & $28.79^{* *}$ & $144.72^{* *}$ \\
\hline $\mathrm{L} 2 \times \mathrm{T} 4$ & $17.62^{* *}$ & 16.89 & $-4.25^{* *}$ & $-6.19^{* *}$ & $7.34^{*}$ & -1.88 & $7.62^{*}$ & $12.73^{* *}$ & $25.14^{* *}$ & $25.16^{* *}$ & $-17.25^{* *}$ & $32.35^{* *}$ & $140.09^{* *}$ \\
\hline $\mathrm{L} 3 \times \mathrm{T} 1$ & $20.73^{* *}$ & $22.62^{*}$ & $-15.62^{* *}$ & $-11.50^{* *}$ & $23.72^{* *}$ & $31.46^{* *}$ & $20.25^{* *}$ & $33.96^{* *}$ & $58.53^{* *}$ & $58.94^{* *}$ & $43.27^{* *}$ & $41.46^{* *}$ & $99.03^{* *}$ \\
\hline $\mathrm{L} 3 \times \mathrm{T} 2$ & $8.48^{* *}$ & $72.86^{* *}$ & $-23.66^{* *}$ & $-19.30^{* *}$ & $15.16^{* *}$ & -1.27 & $14.92^{* *}$ & $75.30^{* *}$ & $99.20^{* *}$ & $100.17^{* *}$ & $41.30^{* *}$ & $44.80^{* *}$ & -6.84 \\
\hline $\mathrm{L} 3 \times \mathrm{T} 3$ & $9.50^{* *}$ & 12.99 & -1.39 & $-3.64^{*}$ & $26.29^{* *}$ & 8.46 & $11.15^{* *}$ & $30.84^{* *}$ & $45.33^{* *}$ & $46.60^{* *}$ & $19.47^{* *}$ & -1.63 & $101.62^{* *}$ \\
\hline
\end{tabular}




\begin{tabular}{|c|c|c|c|c|c|c|c|c|c|c|c|c|c|}
\hline $\mathrm{L} 3 \times \mathrm{T} 4$ & $16.11^{* *}$ & $-26.69^{* *}$ & $-22.94^{* *}$ & $-12.50^{* *}$ & $42.34^{* *}$ & 5.45 & $8.49^{* *}$ & $20.93^{* *}$ & $27.10^{* *}$ & $27.59^{* *}$ & -1.76 & $-11.32^{* *}$ & $111.63^{* *}$ \\
\hline $\mathrm{L} 4 \times \mathrm{T} 1$ & -1.99 & $21.39^{*}$ & $-13.69^{* *}$ & $-8.57^{* *}$ & $75.90^{* *}$ & $34.18^{* *}$ & $11.57^{* *}$ & $21.46^{* *}$ & $33.21^{* *}$ & $33.74^{* *}$ & $40.51^{* *}$ & $74.00^{* *}$ & $64.29^{*}$ \\
\hline $\mathrm{L} 4 \times \mathrm{T} 2$ & $15.83^{* *}$ & $103.40^{* *}$ & $-6.06^{* *}$ & $-7.55^{* *}$ & $75.28^{* *}$ & $20.12^{* *}$ & $6.93^{*}$ & $-5.70^{*}$ & 0.5 & 0.51 & $40.34^{* *}$ & $76.82^{* *}$ & $73.68^{* *}$ \\
\hline $\mathrm{L} 4 \times \mathrm{T} 3$ & $15.83^{* *}$ & $28.56^{* *}$ & $-9.15^{* *}$ & $-7.84^{* *}$ & $38.69^{* *}$ & $37.82^{* *}$ & $-10.86^{* *}$ & $-31.87^{* *}$ & $-40.87^{* *}$ & $-41.29^{* *}$ & $25.00^{* *}$ & $42.59^{* *}$ & $228.01^{* *}$ \\
\hline $\mathrm{L} 4 \times \mathrm{T} 4$ & $17.77^{* *}$ & -20.1 & $-4.93^{* *}$ & $-3.85^{*}$ & $87.44^{* *}$ & $22.74^{* *}$ & 3.28 & $-31.30^{* *}$ & $-32.01^{* *}$ & $-32.56^{* *}$ & $-3.63^{* *}$ & $33.42^{* *}$ & $236.28^{* *}$ \\
\hline $\mathrm{L} 5 \times \mathrm{T} 1$ & -2.66 & 0.5 & $-13.59^{* *}$ & $-12.38^{* *}$ & 0.52 & $12.69^{* *}$ & $-6.71^{* *}$ & $-10.09^{* *}$ & $-12.84^{* *}$ & $-13.09^{* *}$ & $21.77^{* *}$ & $8.69^{* *}$ & $204.53^{* *}$ \\
\hline $\mathrm{L} 5 \times \mathrm{T} 2$ & $7.38^{* *}$ & -9.48 & $-8.21^{* *}$ & $-11.32^{* *}$ & $19.47^{* *}$ & $-15.63^{* *}$ & -3.67 & $29.62^{* *}$ & $28.73^{* *}$ & $29.28^{* *}$ & $16.54^{* *}$ & $21.97^{* *}$ & $202.82^{* *}$ \\
\hline $\mathrm{L} 5 \times \mathrm{T} 3$ & $7.01^{* *}$ & 1.1 & $-5.11^{* *}$ & $-5.88^{* *}$ & $5.50^{*}$ & $19.83^{* *}$ & $-13.31^{* *}$ & $36.24^{* *}$ & $25.88^{* *}$ & $26.13^{* *}$ & $26.24^{* *}$ & $-7.79 * *$ & $45.77^{* *}$ \\
\hline $\mathrm{L} 5 \times \mathrm{T} 4$ & $8.39^{* *}$ & -1.94 & $-8.41^{* *}$ & $-7.69^{* *}$ & $26.61^{* *}$ & $16.01^{* *}$ & $-4.79^{*}$ & $27.01^{* *}$ & $25.26^{* *}$ & $25.78^{* *}$ & $9.93^{* *}$ & $-5.03^{* *}$ & $50.21^{* *}$ \\
\hline $\mathrm{L} 6 \times \mathrm{T} 1$ & $9.21^{* *}$ & $22.08^{* *}$ & $-11.31^{* *}$ & $-8.68^{* *}$ & $43.04^{* *}$ & $31.57^{* *}$ & 6.4 & $66.81^{* *}$ & $76.56^{* *}$ & $77.88^{* *}$ & 1.15 & $24.81^{* *}$ & -13.05 \\
\hline $\mathrm{L} 6 \times \mathrm{T} 2$ & $18.67^{* *}$ & $-25.84^{*}$ & $-11.11^{* *}$ & $-11.31^{* *}$ & $11.19^{* *}$ & $14.03^{* *}$ & -2.51 & $6.77^{* *}$ & $6.67^{*}$ & $6.79^{* *}$ & $-9.03^{* *}$ & $43.65^{* *}$ & $233.47^{* *}$ \\
\hline $\mathrm{L} 6 \times \mathrm{T} 3$ & $18.35^{* *}$ & 4.47 & -2.81 & $-4.23^{*}$ & $44.65^{* *}$ & $31.70^{* *}$ & $26.84^{* *}$ & $6.51^{* *}$ & $36.10^{* *}$ & $37.20^{* *}$ & $-2.41^{*}$ & $41.78^{* *}$ & -7.37 \\
\hline $\mathrm{L} 6 \times \mathrm{T} 4$ & $16.83^{* *}$ & 0.96 & $5.89^{* *}$ & -0.46 & $80.79^{* *}$ & $20.93^{* *}$ & $14.66^{* *}$ & $13.76^{* *}$ & $27.28^{* *}$ & $27.77^{* *}$ & $-23.27^{* *}$ & $7.25^{* *}$ & $242.63^{* *}$ \\
\hline $\mathrm{L} 7 \times \mathrm{T} 1$ & $27.62^{* *}$ & 16.99 & $-6.03^{* *}$ & $-6.99 * *$ & $54.16^{* *}$ & -1.9 & $37.14^{* *}$ & $40.04^{* *}$ & $89.99^{* *}$ & $91.59^{* *}$ & $41.71^{* *}$ & $41.66^{* *}$ & -18.69 \\
\hline $\mathrm{L} 7 \times \mathrm{T} 2$ & $45.94^{* *}$ & $42.85^{* *}$ & $5.11^{* *}$ & -2.41 & $30.02^{* *}$ & $11.11^{* *}$ & $6.62^{*}$ & $33.60^{* *}$ & $40.78^{* *}$ & $41.50^{* *}$ & $16.58^{* *}$ & $26.29^{* *}$ & $226.77^{* *}$ \\
\hline $\mathrm{L} 7 \times \mathrm{T} 3$ & $55.51^{* *}$ & 3.75 & $4.32^{*}$ & -0.62 & $15.93^{* *}$ & $12.82^{* *}$ & $-20.70^{* *}$ & $79.52^{* *}$ & $42.32^{* *}$ & $43.58^{* *}$ & $19.73^{* *}$ & $24.12^{* *}$ & -21.77 \\
\hline $\mathrm{L} 7 \times \mathrm{T} 4$ & $32.74^{* *}$ & 19.77 & $8.04^{* *}$ & 1.23 & $14.65^{* *}$ & 2.93 & $-8.81^{* *}$ & $17.45^{* *}$ & 4.16 & 4.24 & -0.16 & $25.49^{* *}$ & -22.73 \\
\hline
\end{tabular}

***: Significant at $P \leq 0.05$ and $P \leq 0.01$, respectively 


\section{Discussion}

Begomoviruses are known for infecting a large quantity of economically important dicot plants globally. The genus Begomovirus belongs to the family Geminiviridae vectored by the whitefly, Bemisia tabaci. In chilli pepper, the chilli leaf curl disease on chilli plant has been reported from India. A strain of Chilli leaf curl virus-Pakistan (ChiLCV-PK) was associated with chilli leaf curl disease in south-east Asia [31,32]. Meanwhile, tomato leaf curl Joydebpur virus, reported from tomato in Bangladesh, was also noticed to be associated with chilli leaf curl disease in India [33]. Till date genome sequence of four begomoviruses infecting chilli have been characterised from India viz., chilli leaf curl virus (ChiLCV), tomato leaf curl New Delhi virus (ToLCNDV), tomato leaf curl Joydebpur virus (ToLCJV) and recently chilli leaf curl Palampur virus (ChiLCPV). Their distinctive symptoms in infected plants are easily recognised. The typical symptoms consist of leaf curling, puckering, rolling, shortening of internodes and petioles, blistering of interveinal areas, thickening and swelling of the veins, older leaves turned out to be leathery and brittle, crowding of leaves and stunting of whole plants [14]. Moreover, the typical leaf curl symptoms and an increase in disease severity in infected plants are due to the presence of cognate betasatellites associated with the virus [34].

Identification of resistance sources is of utmost importance in any resistant breeding program. Keeping this in mind, natural field screening seemed best to eliminate the genotypes, which showed visible susceptible reaction under natural epiphytotic conditions [35]. The success of disease resistance breeding depends on the genetic variability and the reliable evaluation tests employed for identification of the resistant sources [36]. It is essential to use the most credible tests of resistance when dealing with destructive diseases like ChiLCV. In this direction, various methods have been employed to screen Capsicum germplasm for resistance to ChiLCV viz., screening under natural epiphytotic conditions and artificial inoculation (grafting inoculation and whitefly mediated inoculation) [37].

Breeding for ChiLCV resistance was started in the late sixties in India and natural field screening was mostly used to identify resistance sources based on disease incidence and severity. We, therefore, employed both natural and artificial screening methods in our research. In the present investigation, following graft inoculation, all ten genotypes showed symptom development. Further, these genotypes were confirmed for the presence of virus by amplification of $560 \mathrm{bp}$ DNA fragment specific to the viral genome. Though the virus is present in all the graft inoculated plants, testers showed some level of resistance. Whereas under filed conditions all the four testers were symptomless and among seven lines, two were moderately resistant and the remaining five were moderately susceptible. Previously, Bhutia et al. (2015) reported the minimum per cent disease index (PDI) of 9.22\% [38]. Kumar et al. [39] screened 321 chilli genotypes under field conditions and identified only four genotypes with a highly tolerant reaction against ChiLCV. Whereas, the sixty germplasm lines of Capsicum annuum L., one each of $C$. chinense, C. chacoense and C. baccatum and two of $C$. frutescens were screened and, none of the genotypes was found to be free from the disease[35].

Natural whitefly-mediated inoculation is the most commonly used technique which does not alter the natural virus-vector-host relationships but it's very difficult to control inoculum pressure [40]. In the present experiment, seventy genotypes were screened under natural disease conditions. The phenotypic observations suggested that the chilli plants infected at an early stage remained severely stunted. Their terminal and axillary shoots tend to stay erect, and their leaflets were reduced in size and abnormally shaped. Moreover, a wide variation in the leaf curl virus 
symptoms variability was noticed under natural field conditions. Establishment of advanced symptoms and in the severe form points out that the disease is present in epidemic form and field experiment for ChiLCV screening was successful. [41]. The differential response of genotypes to ChiLCV incidence and symptom expression could be attributed to the fact that the disease incidence and its spread are influenced by the occurrence and population dynamics of the vector whitefly and the weather conditions in the agro-ecosystem [42].

We found a significant difference among the parents and their hybrids for all characters studied. Naresh et al. [43] earlier reported significant differences among chilli genotypes. Significant differences among genotypes were reported also reported by Rodrigues et al. [44] and Geleta and Labuschagne [45] for various fruit and yield traits in chilli pepper. Predominating role of the nonadditive gene action makes it difficult to gather desirable genes because these genes are not fixed in the population [46]. Through the line $\times$ tester analysis, Payakhapaab et al. [47] found significant difference due to crosses and line $\times$ testers for plant height, fruits plant ${ }^{-1}$, fruit weight, yield, fruit length and fruit width. The inheritance of these characters were apparently due to both additive and non-additive gene action. Singh et al. [48] observed the predominance of additive gene effects for fruit weight, fruit width, fruit length and days to flowering. Naresh et al. [43] reported that the mean sum of squares due to genotypes, parents and hybrids, and parent vs hybrids were highly significant for fruit length, fruit width, dry yield plant ${ }^{-1}$ and total carotenoids. Similar to our results of high values of GCA and SCA. High GCA and SCA effects were observed for plant height, days to flower, fruit length, fruit girth, fruit weight, and fruits per plant by various authors [49-51].

The traits with a lower GCA/SCA ratio values hold a non-additive genetic control. Whereas, the characters with a higher GCA/SCA ration possess an additive genetic control [26,52]. Our results indicated the possibility of the exploitation of heterosis along with picking up of superior segregants (pedigree method) in these crosses, especially for fruit yield important under ChiLCV, indicating the importance of exploiting hybrid vigour in these cross combinations.

Krishnamurthy et al. [53] crossed five lines with 30 testers in line $\times$ tester mating design to develop 150 F1 hybrids, and they estimated the extent of mid-parent heterosis. For fruit yield plant-1 (green), seven hybrid combinations showed positive and significant mid-parent heterosis. Moreover, our results are in line with the previous study in tomato performed for the determination of heterosis in tomato under leaf curl virus infestation $[54,55]$.

Overall, the four testers were highly resistant under artificial inoculated conditions. Moreover, lines vs testers showed significant differences for all the characters except for plant height. This suggested considerable differences exist among genotypes, i.e. parents (lines and testers) and their 28 F1 hybrids. The hybrids viz., L3 × T2, L7 × T1, L1 × T1, L6 × T3, L1 × T4, L4 × T2 and L7 × T3 were the most promising with desirable SCA effects, heterosis and per se performance for yield and quality attributes and they were moderately resistant to ChiLCV. The ChiLCV resistance could be improved through recombinant breeding or recurrent selection.

\section{Materials and Methods}

\subsection{Plot Location, Soil and Weather Parameters}

The present investigation was carried out at the Department of Vegetable Science, College of Agriculture, Kerala Agricultural University, Vellayani, India, during 2015-2018. The experimental site was located at $8.50^{\circ}$ North-latitude and $76.90^{\circ}$ East-longitude, at an altitude of $29.00 \mathrm{~m}$ above mean sea level. The soil properties were determined based on the methods defined elsewhere [56] and are presented in Table 10. Whereas, the climate during the entire crop season is presented in Figure 2.

Table10. Physicochemical properties of soil of the experimental field. 


\begin{tabular}{ccc}
\hline Parameter & Value & Rating \\
\hline $\mathrm{pH}$ & 5.60 & Moderately acid \\
\hline Electrical conductivity $\left(\mathrm{dSm}^{-1}\right)$ & 0.074 & Normal \\
\hline Organic carbon $(\%)$ & 1.10 & Medium \\
\hline Available P $\left(\mathrm{kg} \mathrm{ha}^{-1}\right)$ & 43.20 & High \\
\hline Available K & 405.00 & Deficient \\
\hline Exchangeable Ca $(\mathrm{ppm})$ & 250.00 & Deficient \\
\hline Exchangeable Mg $(\mathrm{ppm})$ & 60.00 & Sufficient \\
\hline Available S $(\mathrm{ppm})$ & 25.20 & Sufficient \\
\hline Available Fe (ppm) & 26.60 & Sufficient \\
\hline Available Mn (ppm) & 39.30 & Sufficient \\
\hline Available Zn (ppm) & 6.50 & Sufficient \\
\hline Available Cu (ppm) & 0.52 & \\
\hline
\end{tabular}




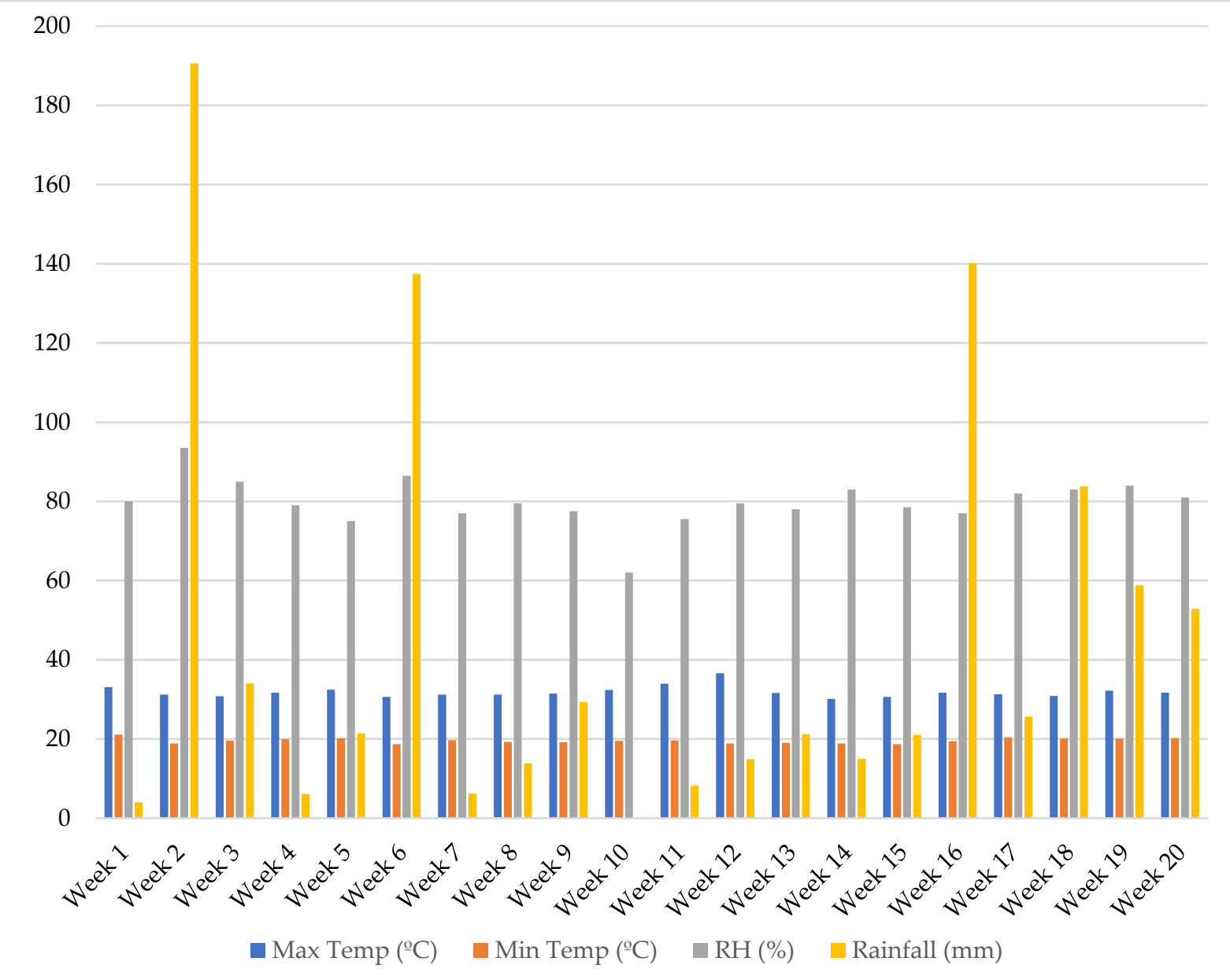

Figure 2. Weather parameters during the entire crop season.

\subsection{Plant Material}

Seven female parents (lines) were crossed with the four testers (male parent) to produce twenty-eight F1 hybrids. The list of genotypes and their source of origin is given in Table 11. For the present investigation, the eleven parental genotypes (lines and testers) were selected based on their yield and ChiLCD disease indices in 2016. The lines were used as the female parents and were crossed with four highly resistant male parents as testers in a line $\times$ tester mating design to produce 28 one-way (excluding reciprocals) $F_{1}$ hybrids. The hand emasculation technique was used to produce the hybrids. The well-matured flower buds which are likely to anthesis next morning were selected and were carefully emasculated during evening time by using forceps and closed with butter paper bags. These emasculated flower buds act as the female parent. The pollens were collected from the male parent of fully matured flowers. On next morning between 8.00 am to $10.00 \mathrm{am}$ (anthesis time of chilli pepper), the emasculated flower buds were pollinated by using male pollens and they were again covered with butter paper bags and labelled. The seeds were collected separately from successfully crossed red ripe fruits. 
The seeds were sown in portrays by using the potting mixture. During summer season thirtyday old healthy seedlings (8-10 cm height) were transplanted in the summer season (MarchMay). The package and practices were followed, as described elsewhere [57]. The experiment was laid out as in a randomized complete block design (RCBD) as three replication. There were twenty plants in each replication. The plant to plant and row to row spacing was $45 \mathrm{~cm}$. However, to facilitate the attack of leaf curl virus disease in the experiment, plant protection measures were not used.

Table 11. Details of parents used in $\mathrm{L} \times \mathrm{T}$ analysis.

\begin{tabular}{ccc}
\hline Name of Parents & Accession Number & Source \\
\hline Lines (female) & & \\
\hline L1 & CHIVAR-3 & IIVR, Varanasi \\
\hline L2 & CHIVAR-7 & IIVR, Varanasi \\
\hline L3 & CHIVAR-6 & IIVR, Varanasi \\
\hline L4 & CA-32 & UAS, Dharwad \\
\hline L5 & Vellayani Athulya & KAU, Thrissur \\
\hline L6 & Keerthi & KAU, Thrissur \\
\hline L7 & CHIVAR-10 & IIVR, Varanasi \\
\hline Testers (male) & & \\
\hline T1 & VLY-09 & KAU, Thrissur \\
\hline T2 & VLY-11 & KAU, Thrissur \\
\hline T3 & VLY- 14 & KAU, Thrissur \\
\hline T4 & CHIVAR-1 & IIVR, Varanasi
\end{tabular}

*IIVR- Indian Institute of Vegetable Research, Varanasi, KAU-Kerala Agricultural University, Thrissur. University of Agricultural Science (UAS), Dharwad.

\subsection{Artificial and Field Screening Against ChiLCV}

Before crossing in order to determine the resistance potential of parental genotypes, the parental genotypes (lines and testers) were subjected to the artificial inoculation methods, namely whitefly mediated inoculation and graft inoculation technique. For the artificial inoculation, chilli seeds were sown in the plug trays filled with vermicompost and cocopeat in 1:1 proportion. The trays were kept in the insect-proof cage. Twenty days after sowing the seedlings were gently removed and transplanted into the plastic pot of size $14 \times 10 \times 13.5 \mathrm{~cm}$ filled with soil mixture with vermicompost and kept in an insect-proof cage for inoculation. For feed acquisition of virus by the whiteflies, ChiLCV infected plant branches were inserted inside the bottles which contain non-viruliferous whiteflies. These flies were allowed to feed on the ChiLCV infected branches for 24 hours (Acquisition period). The healthy chilli seedlings (resistant lines) were inoculated at two-true leaf stage. Cage or single plant inoculation technique was followed for artificial whitefly inoculation $[40,58]$.

Whereas, the graft inoculation was performed as the healthy test plants were grown in pots under insect-proof cage [59]. Small branches $(10-15 \mathrm{~cm})$ were selected from $70-80$ days old test plants and were used for preparing the scions. For rootstock purpose, the ChiLCV infected 
plants were uprooted and transplanted into clay pots and kept under greenhouse conditions (Figure 2). The base of the scion was trimmed to a wedge shape and inserted into a cleft made on the stem of the infected chilli rootstock plant. The graft was then tied firmly using a para film strip (Figure 2). To increase grafting success the plastic zip-lock pouch bags $\left(10^{\prime \prime} \times 12^{\prime \prime}\right.$ inch) were covered over grafted plants (Figure 2).

The presence of ChiLCV from the infected plants was confirmed by PCR employing the degenerate primers [60]. The genotypes after graft inoculation were assessed for presence/absence of viral nucleic acid by Polymerase Chain Reaction (PCR) using a universal degenerate primer (AV494/AC1048) for identification of Geminivirus isolates (subgroup III) [60].
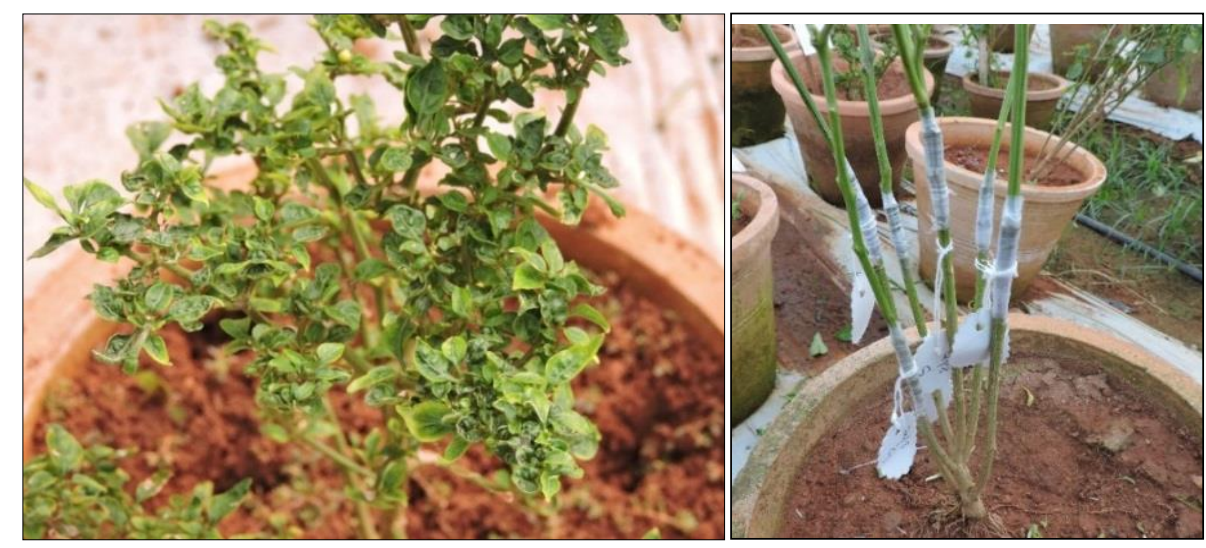

Figure 3. A susceptible plant used as rootstocks (left) and the grafted plant as scion on the infected rootstock (right).

The parents and their hybrids were screened against ChiLCV resistance under natural epiphytotic conditions during 2017-2018. No plant protection measures were provided. The visual observation of the leaf curl incidence (\%) was first recorded 15 days after transplanting. Subsequent observations were recorded at fortnightly intervals, as described by Muniyappa et al. [61]. Five plants were randomly selected from each treatment and the observation was taken at the fortnightly interval after transplanting (DAT) based on the scale defined in detail elsewhere [62]. The specific disease reaction was assigned for all the genotypes based on the coefficient of infection (CI) as suggested by Kumar et al. [63]. The scale used for the scoring of ChiLCV reaction is presented in Table 12.

Table 12. The scale employed for scoring ChiLCV reaction.

\begin{tabular}{cccc}
\hline Symptom & $\begin{array}{c}\text { Severity } \\
\text { grade }\end{array}$ & $\begin{array}{c}\text { Coefficient } \\
\text { of infection } \\
\text { (CI) }\end{array}$ & $\begin{array}{c}\text { Disease reaction } \\
\text { (DR) }\end{array}$ \\
\hline Absence of visual symptom & 0 & 0 & $\begin{array}{c}\text { Symptomless - } \\
\text { SL }\end{array}$ \\
\hline Clearing and curling of top leaves, 0- & 1 & $0.1-5$ & Highly Resistant - \\
$5 \%$ curling & & & HR \\
\hline
\end{tabular}




\begin{tabular}{|c|c|c|c|}
\hline $\begin{array}{l}\text { Clearing of leaves and veins swelling, } \\
6-25\end{array}$ & 2 & $5.1-10$ & Resistant - R \\
\hline \multicolumn{4}{|l|}{$\%$ curling } \\
\hline $\begin{array}{l}\text { Yellowing and puckering of leaves and } \\
\text { veins swelling, } 26-50 \% \text { \% leaf curling }\end{array}$ & 3 & $10.1-20$ & $\begin{array}{l}\text { Moderately } \\
\text { resistant - MR }\end{array}$ \\
\hline $\begin{array}{l}\text { Curling of leaves, internodes blistered } \\
\text { and stunted plant growth, 51-75 \% leaf } \\
\text { curling }\end{array}$ & 4 & $20.1-40$ & $\begin{array}{c}\text { Moderately } \\
\text { susceptible-MS }\end{array}$ \\
\hline $\begin{array}{l}\text { Small deformed leaves, overall stunted } \\
\text { plant growth with very few or no } \\
\text { flowers and fruits, }>75 \% \text { leaf curling }\end{array}$ & 5 & $40.1-70$ & Susceptible - S \\
\hline $\begin{array}{l}\text { Deformed very small leaves, severely } \\
\text { stunted plant growth. The flowers and } \\
\text { fruits were completely absent. }\end{array}$ & 6 & $70.1-100$ & $\begin{array}{c}\text { Highly susceptible } \\
\text { - HS }\end{array}$ \\
\hline
\end{tabular}

The degree of resistance was measured by individual plant score, or individual plant belongs to each class of score (0-6). DSI (Disease severity index) gives the provides an important information regarding the disease reaction of an individual [64]. Percent disease incidence $(\mathrm{PDI}, \%)$ was estimated as the ratio of total infected plant number to the total number of plant $\times 100$. The disease severity index (DSI,\%) was determined 100 (Sum of infected plants in each class/ Total number of plants $\times$ Maximum disease score). Whereas, the coefficient of infection $(\mathrm{CI}, \%)$ was determined based on the formula $(\mathrm{DSI} \times \mathrm{DI}) / 100$.

\subsection{Plants Characterization and Data Analysis}

Observations were recorded from five randomly selected plants to determine the plant characteristics as discussed below.

\subsubsection{Plant Height (cm)}

Plant height was determined at before final harvest from five random plants.

\subsubsection{Primary Branches Plant ${ }^{-1}$}

At the end of the final harvest, the primary branches emerging from the main stem were recorded.

\subsubsection{Days to First Flowering}

From five randomly selected plants, the duration (days) taken to first flower opening from the date of transplanting were calculated and the mean worked out.

\subsubsection{Days to First Harvest}

The days taken from the date of transplanting to the first fruit harvest from five randomly plants were noted and the mean worked out. 


\subsubsection{Fruit Length (cm)}

Ten matured green fruits were randomly selected from each tagged plant in the third harvest. The fruit length $(\mathrm{cm})$ was measured from pedicel attachment of the fruit to its tip end and the mean was worked out.

\subsubsection{Fruit Girth (cm)}

The girth of fruit was noted from the central fruit by using twine and scale. The same fruits which were used to measure length of fruit was used to measure fruit girth, and the average girth was noted.

\subsubsection{Fruits Plant ${ }^{-1}$}

The mature fruits number from each harvest were counted and recorded. Counted fruits were added and the average number of fruits per plant was worked out.

\subsubsection{Fruit Weight ( $g$ )}

The average weight of fruits was measured from 10 randomly picked fruits from third picking. The weight of fruits was measured on an electronic balance.

\subsubsection{Yield Plant ${ }^{-1}(g)$}

Yield plant ${ }^{-1}$ was computed by adding the mature green fruit weight from every harvest and dividing by the number of randomly selected plants (five); the mean weight is expressed in grams.

\subsubsection{Yield $\operatorname{Plot}^{-1}\left(\mathrm{~kg} / 6.48 \mathrm{~m}^{2}\right)$}

From each plot, harvested weight of fruits was calculated and expressed in kilograms.

\subsubsection{Vitamin C ( $m g 100 g^{-1}$ fresh fruit weight)}

To estimate the vitamin C content from green fruits, 2,6-dichloro phenol indophenol dye procedure method was followed $[29,65]$.

4.4.12. Carotenoids ( $m g$ $100 g^{-1}$ )

The carotenoids fraction was determined using UV-visible spectrophotometric measurements at two characteristic wavelengths based on the method explained elsewhere [66]. Briefly, the dried red ripe fruits were selected and were ground into a fine powder. This powder $(100 \mathrm{mg})$ was extracted with acetone $(25 \mathrm{ml})$. This extract was transferred to a volumetric flask and the volume was made up to $50 \mathrm{ml}$ by adding acetone. By using acetone as blank, the absorbance of the sample was recorded at two wavelengths (472 nm and 508 $\mathrm{nm})$.

The Line $\times$ Tester genetic analysis was performed for the determination of GCA, SCA along with the variance components with the help of software program PBTools (PBTools-1.4, 2014) [67]. Heterosis magnitude was identified in relation to mid-parent, and better parent values based on the formula: $100 \times\left(\left(\mathrm{F}_{1}-\mathrm{MP}\right) / \mathrm{MP}\right)$, where $\mathrm{F}_{1}$ is the hybrid mean, and MP is the mean 
of the parents and $100 \times\left(\left(\mathrm{F}_{1}-\mathrm{BP}\right) / \mathrm{BP}\right)$, where $\mathrm{F} 1$ is the hybrid mean and $\mathrm{BP}$ is the mean of the better parent.

Author Contributions: S.I., R.M. and V.S. conceived and designed the project. S.I. and R.M. supervised the study. V.S. performed the experiments. V.S. and P.K. analysed the data. V.S. and P.K. wrote the paper and corrected the final draft. All authors read and approved the final manuscript.

Funding: This research received no external funding.

Acknowledgments: The authors are thankful to the anonymous reviewers for their careful reading of the manuscript and for providing insightful suggestions.

Conflicts of Interest: The authors declare no conflict of interest.

\section{References}

1. Pandey, J.; Srivastava, K.; Kumar, S. Chilli (Capsicum spp.): A Diverse Crop with Innovative Uses. Modern Trends in Microbial Biodiversity of Natural Ecosystem 187.

2. Bosland, P. W.; Votava, E. J. Peppers: Vegetable and Spice Capsicums. Peppers 2012, 2, 1-230.

3. Ramchiary, N.; Kole, C. The Capsicum Genome; Springer, 2019; ISBN 978-3-319-97217-6.

4. Tripodi, P.; Kumar, S. The Capsicum Crop: An Introduction. In; 2019; pp. 1-8 ISBN 978-3-319-97217-6.

5. Zhang, X.; Zhang, Z.; Gu, X.; Mao, S.; Li, X.; Chadœuf, J.; Palloix, A.; Wang, L.; Zhang, B. Genetic diversity of pepper (Capsicum spp.) germplasm resources in China reflects selection for cultivar types and spatial distribution. Journal of Integrative Agriculture 2016, 15, 1991-2001, doi:10.1016/S20953119(16)61364-3.

6. Patel, J. A.; Patel, M. J.; Bhanvadia, A. S.; Acharya, R. R.; Bhalala, M. K. Extent of natural cross pollination with GMS lines in chilli (Capsicum annuum L.); 2001;

7. Prasath, D.; Ponnuswami, V. Heterosis and combining ability for morphological, yield and quality characters in paprika type chilli hybrids. Indian Journal of Horticulture 2008, 65, 441-445.

8. Schweiggert, U.; Carle, R.; Schieber, A. Conventional and alternative processes for spice production-a review. Trends in food science \& technology 2007, 18, 260-268.

9. Wahyuni, Y.; Ballester, A.-R.; Sudarmonowati, E.; Bino, R.; Bovy, A. Secondary Metabolites of Capsicum Species and Their Importance in the Human Diet. Journal of natural products 2013, doi:10.1021/np300898z.

10. Dwivedi, S.; Goldman, I.; Ortiz, R. Pursuing the Potential of Heirloom Cultivars to Improve Adaptation, Nutritional, and Culinary Features of Food Crops. Agronomy 2019, 9, 441, doi:10.3390/agronomy9080441.

11. Berke, T. Hybrid Seed Production in Capsicum. Journal of New Seeds 2000, 1, 49-67, doi:10.1300/J153v01n03_02.

12. Pandey, P.; Irulappan, V.; Bagavathiannan, M. V.; Senthil-Kumar, M. Impact of Combined Abiotic and Biotic Stresses on Plant Growth and Avenues for Crop Improvement by Exploiting Physiomorphological Traits. Front Plant Sci 2017, 8, doi:10.3389/fpls.2017.00537.

13. Leke, W. N.; Mignouna, D. B.; Brown, J. K.; Kvarnheden, A. Begomovirus disease complex: emerging threat to vegetable production systems of West and Central Africa. Agriculture E Food Security 2015, 4, 1, doi:10.1186/s40066-014-0020-2.

14. Thakur, H.; Jindal, S.; Sharma, A.; Dhaliwal, M. Chilli leaf curl virus disease: a serious threat for chilli cultivation. Journal of Plant Diseases and Protection 2018, 125, doi:10.1007/s41348-018-0146-8.

15. Kumar, R. V.; Singh, A.; Chakraborty, Dr. S. A new monopartite begomovirus species, Chilli leaf curl Vellanad virus, and associated betasatellites infecting chilli in the Vellanad region of Kerala, India. New Disease Reports 2012, 25, doi:10.5197/j.2044-0588.2012.025.020. 
16. Rodríguez, E.; Téllez, M. M.; Janssen, D. Whitefly Control Strategies against Tomato Leaf Curl New Delhi Virus in Greenhouse Zucchini. Int J Environ Res Public Health 2019, 16, doi:10.3390/ijerph16152673.

17. Mammadov, J.; Buyyarapu, R.; Guttikonda, S. K.; Parliament, K.; Abdurakhmonov, I. Y.; Kumpatla, S. P. Wild Relatives of Maize, Rice, Cotton, and Soybean: Treasure Troves for Tolerance to Biotic and Abiotic Stresses. Front. Plant Sci. 2018, 9, doi:10.3389/fpls.2018.00886.

18. Vasudevan, K.; Vera Cruz, C. M.; Gruissem, W.; Bhullar, N. K. Large scale germplasm screening for identification of novel rice blast resistance sources. Front Plant Sci 2014, 5, doi:10.3389/fpls.2014.00505.

19. Gallois, J.-L.; Moury, B.; German-Retana, S. Role of the Genetic Background in Resistance to Plant Viruses. Int J Mol Sci 2018, 19, doi:10.3390/ijms19102856.

20. Prashant, K.; Major, S. D.; Salesh, K. J.; Ashutosh, S.; Vikrant, T.; Navjot, S. B.; Mahesh, K. R. Heterosis and leaf curl virus resistance in rainy season tomato under North Indian conditions. African Journal of Agricultural Research 2015, 10, 2763-2772, doi:10.5897/AJAR2014.9133.

21. Kaushik, P.; Dhaliwal, M. S. Diallel Analysis for Morphological and Biochemical Traits in Tomato Cultivated under the Influence of Tomato Leaf Curl Virus. Agronomy 2018, 8, 153, doi:10.3390/agronomy8080153.

22. Sreenivas, M.; Bhattacharjee, T.; Sharangi, A. B.; Maurya, P. K.; Banerjee, S.; Chatterjee, S.; Maji, A.; Mandal, A. K.; Chakraborty, I.; Chattopadhyay, A. Breeding chili pepper for simultaneous improvement in dry fruit yield, fruit quality and leaf curl virus disease tolerance. International Journal of Vegetable Science 2019, 0, 1-30, doi:10.1080/19315260.2019.1648351.

23. Plant Breeding and Crop Production. In Van Nostrand's Scientific Encyclopedia; American Cancer Society, 2007 ISBN 978-0-471-74398-9.

24. Bertan, I.; Costa de Oliveira, A. Parental Selection Strategies in Plant Breeding Programs. 2007.

25. Clark, P. J. Kempthorne, Oscar. An Introduction to Genetic Statistics. John Wiley \& Sons, Inc., N.Y. Pp. xvii + 545. 1957. Price, \$12.75. J Mammal 1958, 39, 313-313, doi:10.2307/1376224.

26. Kaushik, P. Line $\times$ Tester Analysis for Morphological and Fruit Biochemical Traits in Eggplant (Solanum melongena L.) Using Wild Relatives as Testers. Agronomy 2019, 9, 185, doi:10.3390/agronomy9040185.

27. Fasahat, P.; Rajabi, A.; Rad, J. M.; Derera, J. Principles and Utilization of Combining Ability in Plant Breeding. Biometrics \& Biostatistics International Journal 2016, 4, 1-0, doi:10.15406/bbij.2016.4.00085.

28. Teodoro, L. P. R.; Bhering, L. L.; Gomes, B. E. L.; Campos, C. N. S.; Baio, F. H. R.; Gava, R.; Júnior, C. A. da S.; Teodoro, P. E. Understanding the combining ability for physiological traits in soybean. PLOS ONE 2019, 14, e0226523, doi:10.1371/journal.pone.0226523.

29. C. S., A.; S, V.; I, S.; Kaushik, P. Diallel Analysis of Chilli Pepper (Capsicum annuum L.) Genotypes for Morphological and Fruit Biochemical Traits. Plants 2020, 9, 1, doi:10.3390/plants9010001.

30. Vijeth, S.; Dhaliwal, M. S.; Jindal, S. K.; Sharma, A. Evaluation of tomato hybrids for resistance to leaf curl virus disease and for high-yield production. Hortic. Environ. Biotechnol. 2018, 59, 699-709, doi:10.1007/s13580-018-0080-5.

31. Fauquet, C. M.; Bisaro, D. M.; Briddon, R. W.; Brown, J. K.; Harrison, B. D.; Rybicki, E. P.; Stenger, D. C.; Stanley, J. Revision of taxonomic criteria for species demarcation in the family Geminiviridae, and an updated list of begomovirus species. Archives of virology 2003, 148, 405-420.

32. Jones, D. R. Plant viruses transmitted by whiteflies. European Journal of Plant Pathology 2003, 109, $195-219$.

33. Dhaliwal, M. S.; Sharma, A. Breeding for Resistance to Virus Diseases in Vegetable Crops. Innovations in Horticultural Sciences 2016, 303-327. 
34. Singh, A. K.; Chattopadhyay, B.; Chakraborty, S. Biology and interactions of two distinct monopartite begomoviruses and betasatellites associated with radish leaf curl disease in India. Virology journal 2012, 9,43 .

35. Srivastava, A.; Mangal, M.; Saritha, R. K.; Kalia, P. Screening of chilli pepper (Capsicum spp.) lines for resistance to the begomoviruses causing chilli leaf curl disease in India. Crop Protection 2017, 100, 177185.

36. St. Clair, D. A. Quantitative disease resistance and quantitative resistance loci in breeding. Annual review of phytopathology 2010, 48, 247-268.

37. Sastry, K. S.; Zitter, T. A. Plant Virus and Viroid Diseases in the Tropics: Volume 2: Epidemiology and Management; Springer Science \& Business Media, 2014;

38. Bhutia, N. D.; Seth, T.; Shende, V. D.; Dutta, S.; Chattopadhyay, A. Estimation of Heterosis, dominance effect and genetic control of fresh fruit yield, quality and leaf curl disease severity traits of chilli pepper (Capsicum annuum L.). Scientia Horticulturae 2015, 182, 47-55, doi:10.1016/j.scienta.2014.11.017.

39. Kumar, S.; Kumar, R.; Kumar, S.; Kumar Singh, A.; Singh, M.; Bahadur Rai, A.; Rai, M. Incidence of leaf curl disease on Capsicum germplasm under field conditions. Indian Journal of Agricultural Sciences 2011, $81,187$.

40. Picó, B.; Díez, M. J.; Nuez, F. Evaluation of whitefly-mediated inoculation techniques to screen Lycopersicon esculentum and wild relatives for resistance to Tomato yellow leaf curl virus. Euphytica 1998, 101, 259-271, doi:10.1023/A:1018353806051.

41. Tivoli, B.; Baranger, A.; Avila, C. M.; Banniza, S.; Barbetti, M.; Chen, W.; Davidson, J.; Lindeck, K.; Kharrat, M.; Rubiales, D.; Sadiki, M.; Sillero, J. C.; Sweetingham, M.; Muehlbauer, F. J. Screening techniques and sources of resistance to foliar diseases caused by major necrotrophic fungi in grain legumes. Euphytica 2006, 147, 223-253, doi:10.1007/s10681-006-3131-4.

42. Ssekyewa, C.; Damme, P. v.; Nagawa, F.; Kyamanywa, S.; Steele, K. A. Relationship between Tomato Yellow Leaf Curl Viruses and the Whitefly Vector. Journal of Science and Sustainable Development 2010, 3, doi:10.4314/jssd.v3i1.67750.

43. P., N.; V.k., R.; B., L. R.; C., A. R.; V., V.; K., M. R. Genetic analysis for fruit biochemical traits (capsaicinoids and carotenoids) and dry fruit yield in chilli (Capsicum annuum L.). Industrial Crops and Products 2016, 94, 920-931, doi:10.1016/j.indcrop.2016.10.006.

44. Rodrigues, R.; Gonçalves, L.; Bento, C.; Sudré, C.; Robaina, R.; Júnior, A. Combining ability and heterosis for agronomic traits in chili pepper. Horticultura Brasileira 2012, 30, 226-233, doi:10.1590/S010205362012000200008.

45. Geleta, L. F.; Labuschagne, M. T. Estimates of combining ability for agronomic traits in pepper (Capsicum annuum L.). South African Journal of Plant and Soil 2006, 23, 73-77, doi:10.1080/02571862.2006.10634734.

46. Spaldon*, S.; Jabeen, N.; Khan, S. H.; Makdoomi, M. I. Combining ability and gene action in chilli (Capsicum annum L.). Indian Journal Of Agricultural Research 49, 255-259, doi:10.5958/0976058X.2015.00040.2.

47. Payakhapaab, S.; Boonyakiat, D.; Nikornpun, M. Evaluation of heterosis and combining ability of yield components in Chillies. Journal of Agricultural Science (Toronto) 2012, 4, 154-161.

48. Singh, P.; Cheema, D. S.; Dhaliwal, M.; Garg, N. Heterosis and combining ability for earliness, plant growth, yield and fruit attributes in hot pepper (Capsicum annuum L.) involving genetic and 
cytoplasmic-genetic male sterile lines. Scientia Horticulturae 2014, 168, 175-188, doi:10.1016/j.scienta.2013.12.031.

49. Do Rêgo, E. R.; Do Rêgo, M. M.; Finger, F. L.; Cruz, C. D.; Casali, V. W. D. A diallel study of yield components and fruit quality in chilli pepper (Capsicum baccatum). Euphytica 2009, 168, 275-287.

50. Hasanuzzaman, M.; Hakim, M. A.; Fersdous, J.; Islam, M. M.; Rahman, L. Combining ability and heritability analysis for yield and yield contributing characters in chilli (Capsicum annuum) landraces. Plant Omics 2012, 5, 337.

51. Chakrabarty, S.; Islam, A. K. M. A.; Mian, M. A. K.; Ahamed, T. Combining Ability and Heterosis for Yield and Related Traits in Chili (Capsicum annuum L.). The Open Agriculture Journal 2019, 13, doi:10.2174/1874331501913010034.

52. Kaushik, P.; Plazas, M.; Prohens, J.; Vilanova, S.; Gramazio, P. Diallel genetic analysis for multiple traits in eggplant and assessment of genetic distances for predicting hybrids performance. PLOS ONE 2018, 13, e0199943, doi:10.1371/journal.pone.0199943.

53. SL, Dr. K.; Mohan Rao, A.; Reddy, K.; Ramesh, S.; Hittalmani, S.; Rao, M. Limits of parental divergence for the occurrence of heterosis through morphological and AFLP marker in chilli (Capsicum annuum L.). Current science 2013, 104, 738-746.

54. Kaushik, P.; Dhaliwal, M. S.; Jindal, S. K.; Srivastava, A.; Tyagi, V.; Brar, N. S.; Rana, M. K. Heterosis and leaf curl virus resistance in rainy season tomato under North Indian conditions. AJAR 2015, 10, 27632772, doi:10.5897/AJAR2014.9133.

55. KAUSHIK, P. Tomato Leaf Curl Virus Resistance in Tomato (Solanum lycopersicum) Hybrids Grown in the Rainy Season under Punjab Conditions. Arch Insect Biochem Physiol 58, 216-225.

56. Bandyopadhyay, K.; Aggarwal, P.; Chakraborty, D.; Pradhan, S.; Garg, R.; Singh, R. Practical Manual on Measurement of Soil Physical Properties Practical; 2012;

57. Chilli Available online: http://www.celkau.in/Crops/Vegetables/Chilli/chilli.aspx (accessed on Jan 12, 2019).

58. Inoue-Nagata, A. K.; Nagata, T.; Ávila, A. C. de; Giordano, L. de B. A reliable begomovirus inoculation method for screening Lycopersicon esculentum lines. Horticultura Brasileira 2007, 25, 447-450, doi:10.1590/S0102-05362007000300024.

59. Bedford, I. D.; Briddon, R. W.; Brown, J. K.; Rosell, R. C.; Markham, P. G. Geminivirus transmission and biological characterisation of Bemisia tabaci (Gennadius) biotypes from different geographic regions. Annals of Applied Biology 1994, 125, 311-325, doi:10.1111/j.1744-7348.1994.tb04972.x.

60. Wyatt, S. D.; Brown, J. K. Detection of subgroup III geminivirus isolates in leaf extracts by degenerate primers and polymerase chain reaction. PHYTOPATHOLOGY 1996, 86, 1288-1293.

61. Muniyappa, V.; Jalikop, S. H.; Saikia, A. K.; Shivashankar, G.; Bhat, A. I.; Ramappa, H. K. Reaction of Lycopersicon cultivars and wild accessions to tomato leaf curl virus. Euphytica 1991, 56, 37-41.

62. Banerjee, M. K.; Kalloo, M. K. Sources and inheritance of resistance to leaf curl virus in Lycopersicon. Theoretical and Applied Genetics 1987, 73, 707-710.

63. Kumar, S.; Kumar, S.; Singh, M.; Singh, A. K.; Rai, M. Identification of host plant resistance to pepper leaf curl virus in chilli (Capsicum species). Scientia Horticulturae 2006, 110, 359-361, doi:10.1016/j.scienta.2006.07.030.

64. Pyne, R. M.; Koroch, A. R.; Wyenandt, C. A.; Simon, J. E. Inheritance of Resistance to Downy Mildew in Sweet Basil. Journal of the American Society for Horticultural Science 2015, 140, 396-403,

doi:10.21273/JASHS.140.5.396. 
65. Manju, P. R.; Sreelathakumary, I. Quality parameters in hot chilli ( Capsicum chinense Jacq.). Journal of Tropical Agriculture 2006, 40, 7-10.

66. Topuz, A.; Ozdemir, F. Assessment of carotenoids, capsaicinoids and ascorbic acid composition of some selected pepper cultivars (Capsicum annuum L.) grown in Turkey. Journal of Food Composition and Analysis 2007, 20, 596-602.

67. Products-Quantitative Genetics and Biometrics Cluster Available online: http://bbi.irri.org/products (accessed on Dec 13, 2018). 